\title{
Connecting electroweak-scale observables to BSM physics through EFT and Bayesian statistics
}

\author{
Anisha $\odot,{ }^{1, *}$ Supratim Das Bakshi $\odot,{ }^{1, \dagger}$ Joydeep Chakrabortty, ${ }^{1, \hbar}$ and Sunando Kumar Patra ${ }^{2, \S}$ \\ ${ }^{1}$ Department of Physics, Indian Institute of Technology, Kanpur-208016, India \\ ${ }^{2}$ Department of Physics, Bangabasi Evening College, 19 Rajkumar Chakraborty Sarani, \\ Kolkata 700009, West Bengal, India
}

(Received 18 December 2020; accepted 22 February 2021; published 22 April 2021)

\begin{abstract}
Recognizing the potential of effective field theories to posit multiple beyond Standard Model (BSM) scenarios in a similar footing with a possibility to compare them, we inspect the effects of 11 single scalar-multiplet extensions of the SM on the combined set of electroweak precision observables and Higgs signal strength data by systematically integrating out the heavy multiplets and computing the resulting Standard Model effective field theory operators and Wilson coefficients (WCs) up to oneloop level. Noting that multiple BSM models give rise to a degenerate set of WCs, we then perform Bayesian statistical inference both directly on the BSM parameters and on the associated set of independent WCs. Using the posteriors of the BSM parameters, we infer the respective (correlated) WC distributions and compare both the model-independent and -dependent analyses by overlaying the 2D marginal WC posteriors from both processes, thus laying the groundwork for a data-driven attempt to compare diverse BSM theories of different origins, and hopefully, a possible way to approach the intractable inverse problem. We also demonstrate, with an example model, the crucial role of theoretical constraints to rule out large chunks of BSM parameter spaces. All numerical results are available on GitHub.
\end{abstract}

DOI: 10.1103/PhysRevD.103.076007

\section{INTRODUCTION}

Despite the immense success of the Standard Model (SM), it is still inadequate to explain a plethora of phenomena in the high-energy physics spectrum. There has been no direct evidence of any beyond Standard Model (BSM) physics after the discovery of the Higgs boson. We thus need to refer to indirect evidence hinting toward BSM scenarios. Among the observables with the potential to constrain BSM physics and thus to act as indirect evidence for new physics (NP), electroweak precision observables (EWPO) and those from Higgs data play an important role. To effectively use these observables to constrain BSM parameter space, we need to bridge the gap between any BSM physics residing at a

\footnotetext{
*anisha@iitk.ac.in

†sdbakshi@iitk.ac.in

*joydeep@iitk.ac.in

sunando.patra@gmail.com
}

Published by the American Physical Society under the terms of the Creative Commons Attribution 4.0 International license. Further distribution of this work must maintain attribution to the author(s) and the published article's title, journal citation, and DOI. Funded by SCOAP ${ }^{3}$. high scale and the observables lying at low energy. The Standard Model effective field theory (SMEFT) $[1,2]$ links the BSM theories to the low-energy observables using the higher-dimension operators originating from integrating out the heavy degrees of freedom (d.o.f.). The SMEFT effective operators, for a given mass dimension and defined using particle content and symmetry of the SM, offer additional contributions to the SM predictions of the low-energy observables. These modifications are recast in terms of the Wilson coefficients (WCs) that carry the footprints of the unknown NP.

There have been numerous works over the years to constrain the SMEFT WCs of dimension-six operators in a model-independent manner. The general strategy has been to perform statistical inference on these WCs using the available data, either taking one of them at a time or all of the pertinent WCs (to which the data are sensitive) together. The SMEFT operators are frequently discussed in the Warsaw [2] and SILH [3-5] bases. The inferences are drawn mostly in a frequentist framework [6-18], though some Bayesian analyses have been done as well [19-22]. The main idea here is that once a BSM theory is matched to SMEFT, the bounds on the WCs can be converted to that of the BSM parameters.

A lot of work has been done to match various BSM theories to the SMEFT (up to one-loop order) [23-32], 
enabling the community to express the SMEFT Wilson coefficients, and in turn, the low-energy observables (EWPO, Higgs signal strengths, etc.) in terms of the BSM parameters. Some (model-dependent) global fits have also been done to constrain specific BSM parameters from these matching results $[7,15,33-36]$. The main caveats of this yet-accepted process are twofold: First, not all WCs are modified within the scope of a specific model and even for those which are affected, the effects are not of the same degree; i.e., not all WCs are similarly sensitive to all model parameters. This set of pertinent WCs also varies with chosen BSM models. Second, though the model-independent inferences performed on WCs can point to a conservative estimate of the BSM parameter space, in reality, they are often highly nonlinear functions of these parameters, and the actual parameter space (obtained from a direct inference on the parameters themselves) may differ a lot from the modelindependent estimates.

The motivation of this work is thus to probe the relative capacity of these model-independent analyses to predict the BSM parameter spaces, in comparison with direct inference done on the parameters themselves. The main challenge in this endeavor is to obtain the SMEFT WCs in terms of the BSM parameters. We use the Mathematica package CODEx [37] to this end. Given the BSM Lagrangian, CODEX can provide a list of the different dimension-six operators and their corresponding WCs at one loop, in terms of different BSM model parameters. For statistical inference, we choose the Bayesian framework, and all required analyses are performed using the Mathematica package optEx [38].

In this article, we work with the Warsaw basis (a complete basis) of dimension-six SMEFT operators, 18 of which affect the data (EWPO and Higgs signal strength) considered in this analysis. The relevant $18 \mathrm{WCs}$ fit is performed in a model-independent manner in two ways: taken all together and taken one at a time. We also consider 11 BSM scenarios, which after matching up to one loop, ${ }^{1}$ generate a subset of the dimension-six operators along with the corresponding WCs expressed as nonlinear functions of the respective BSM parameters. Out of the 18 relevant operators contributing to the observables, only ten operators are collectively generated by these BSM models. So, we show the ten WCs fit also.

While studying the allowed parameter space of a set of specific WCs, we are interested in distinguishing the case where they are independent from the ones where they are correlated. These correlations are a result of their dependence on the model parameters. Matching each of these BSMs with SMEFT, we generate the effective operators and their corresponding WCs in terms of the BSM parameters. We perform a fit on the set of WCs generated

\footnotetext{
${ }^{1}$ We consider one-loop processes for the heavy field propagator in the loop (pure heavy loop) only.
}

for each BSM scenario without including the relations of WCs with the respective model parameters, i.e., by treating WCs to be free parameters. Furthermore, using the matching results, the WCs generated in a specific BSM scenario and expressed in terms of the respective model parameters lead to the direct statistical bounds on the BSM parameters from the observables considered. Using the bounds on the model parameters, the parameter space of the WCs is again explored, which is obtained to be more constrained and thus exhibits the model-dependent allowed parameter space of the WCs. In this way, a comparison of the allowed WCs space of the independent WCs and the correlated WCs is shown for a given BSM.

The work is organized as follows: Sec. II introduces the observables relevant to the present analysis. In Sec. III, we discuss the SMEFT contributions to these observables and perform a model-independent analysis using the relevant WCs. In Sec. IV, we introduce 11 BSM scenarios with the potential to affect the observables in this analysis and obtain individual statistical inferences on each of them. In Sec. V, we compare the model-independent and -dependent results obtained in the previous two sections by inspecting the WC space populated by these results. Numerical results of the entire analysis (including those not included in the draft) are available in the GitHub repository [39] associated with this work.

\section{THE OBSERVABLES $\left(\mathcal{O}_{i}^{\prime} s\right)$}

As mentioned before, the chosen set of observables for both model-dependent and -independent analyses in the present work are the EWPO and Higgs signal strengths. We summarize both the experimental inputs and the SM expressions of the observables in this section.

\section{A. Electroweak precision observables}

The EWPO under consideration for our analysis includes the higher-order radiative corrections which are parametrized in terms of the five SM parameters: $Z$-boson mass $\left(m_{Z}\right)$, Higgs mass $\left(m_{H}\right)$, top quark mass $\left(m_{t}\right)$, strong coupling constant $\left[\alpha_{s}\left(m_{Z}^{2}\right)\right]$, and hadronic contributions to the running of $\alpha\left[\Delta \alpha_{\text {had }}^{(5)}\left(m_{Z}^{2}\right)\right]$. As experimental inputs, we have used (i) EWPO measured at the $Z$-mass pole [14] and their correlations [40] and (ii) mass and decay width of $W$ [41]. Some more details on these corrections to the EWPO are listed below.

(i) $\sin ^{2} \theta_{\text {eff }}^{l}$ receives up to full two-loop electroweak, partial three-loop and four-loop QCD corrections; see Refs. $[14,42]$. The missing higher-order correction is estimated to be $4.7 \times 10^{-5}$, included as theoretical uncertainty in the computation.

(ii) Partial decay width ratios and hadronic peak cross section of $Z$ receives up to full two-loop fermionic corrections; see Refs. [14,43]. 
TABLE I. The Higgs signal strengths from both ATLAS and CMS.

\begin{tabular}{|c|c|c|}
\hline \multicolumn{2}{|c|}{ Higgs signal strengths } & References \\
\hline 7 and $8 \mathrm{TeV}$ Run-I data & Combined ATLAS and CMS measurements & Table 8 of Ref. [47] \\
\hline & Combined ATLAS and CMS measurement of $\mu_{p p}^{\mu \mu}$ & Table 13 of Ref. [47] \\
\hline & ATLAS measurement of $\mu_{p p}^{Z \gamma}$ & Figure 1 of Ref. [48] \\
\hline \multirow[t]{7}{*}{$13 \mathrm{TeV}$ ATLAS Run-II data } & $H \rightarrow Z Z^{*}$ at $139 \mathrm{fb}^{-1}$ & Table 8 of Ref. [49] \\
\hline & Measurement of $\mu_{p p}^{Z \gamma}$ at $139 \mathrm{fb}^{-1}$ & Ref. [50] \\
\hline & Measurement of $\mu_{p p}^{\mu \mu}$ at $139 \mathrm{fb}^{-1}$ & Ref. [51] \\
\hline & $V H \rightarrow H \rightarrow b \bar{b}$ at $139 \mathrm{fb}^{-1}$ & Ref. [52] \\
\hline & $\begin{array}{l}\text { Measurements for Higgs production through gluon } \\
\text { and vector boson fusions at } 80 \mathrm{fb}^{-1}\end{array}$ & $\begin{array}{l}\text { Figure } 5 \text { of Ref. [53] (correlations } \\
\text { in Fig. 6) }\end{array}$ \\
\hline & Associated production of a Higgs boson with $t \bar{t}$ & Refs. $[54,55]$ \\
\hline & $V H \rightarrow H \rightarrow W W^{*}$ at $36.1 \mathrm{fb}^{-1}$ & Ref. $[56]$ \\
\hline \multirow[t]{2}{*}{$13 \mathrm{TeV}$ CMS Run-II data } & Signal strengths data up to $35.9 \mathrm{fb}^{-1}$ & $\begin{array}{l}\text { Table } 3 \text { of Ref. [57] (correlations } \\
\text { in auxiliary material) }\end{array}$ \\
\hline & Measurements of $\mu_{Z H}^{c c}$ and $\mu_{W H}^{c c}$ & Ref. [58] \\
\hline
\end{tabular}

(iii) $Z$ pole asymmetry observables estimated using $\sin ^{2} \theta_{\text {eff }}^{f}{ }^{2}$; see Refs. [14,44].

(iv) Mass of the $W$ boson receives up to two-loop complete and four-loop QCD corrections; see Refs. [14,45].

(v) Decay width of the $W$ boson receives up to one-loop electroweak corrections; see Refs. $[14,46]$.

\section{B. Higgs signal strengths}

The Higgs signal strengths used in our analysis contain the latest Run-I and -II LHC data. The details of the relevant experimental inputs are tabulated in Table I.

\section{MODEL-INDEPENDENT ANALYSIS}

\section{A. SMEFT contributions to the observables}

The SMEFT induces corrections to the fit observables capturing the new physics lying beyond the cutoff scale $(\Lambda)$ of the EFT. We consider the SMEFT contributions to these observables from dimension-six effective operators (in the Warsaw basis) [2]. The EWPO and the Higgs observables can be expressed in terms of the associated WCs $\left(\mathcal{C}_{i}\right)$ as

$$
\mathcal{O}_{\mathrm{NP}}=\mathcal{O}_{\mathrm{SM}}+\sum_{i} \frac{\mathcal{A}_{i}}{\Lambda^{2}} \mathcal{C}_{i}
$$

where $\mathcal{O}_{\mathrm{NP}}$ represents the expressions of observables after including the SMEFT dimension-six operator corrections, and $\mathcal{O}_{\mathrm{SM}}$ represents the SM expressions for the observables discussed in Sec. II. The $\mathcal{A}_{i}$ 's are functions of the five SM parameters (see Sec. II A), and $i$ runs over the number of dimension-six operators pertinent to the observables in question (18 in this work; see Table II). The WCs

\footnotetext{
${ }^{2} \theta_{\text {eff }}^{f}$, the effective Weinberg mixing angle, receives the corrections from fermions only.
}

encapsulate the effect of the NP on top of the SM estimates. Contributions from the SMEFT operators to the observables are linear in order and discussed below.

The SM expressions of the EWPO are modified by the effective operators: $Q_{H}, Q_{H D}, Q_{H \square}, Q_{H W B}, Q_{H l}^{(1)}, Q_{H l}^{(3)}$, $Q_{H e}, Q_{H q}^{(1)}, Q_{H q}^{(3)}, Q_{H u}, Q_{H d}$, and $Q_{l l}$ through the redefinitions of the fields and the couplings. These modifications to the EWPO are captured through the corrections in $\alpha, m_{Z}$, and $G_{F}$ [11,59]. Following the guidelines in Refs. [11, 59-61], the corrections to the EWPO in the presence of these SMEFT operators are calculated. The modification of the theoretical predictions of the Higgs boson production and decay rates due to SMEFT operators (in the SILH-like basis) are discussed in Ref. [62], which we rewrite in terms of dimension-six operators in the Warsaw basis (for the operator basis translation, see [60]). Following Ref. [62], we only consider the contributions from the third generation of fermions for the operators $Q_{e H}, Q_{u H}$, and $Q_{d H}$.

\section{B. Statistical inference}

Adopting the Bayesian framework, all inferences throughout this work are obtained by sampling the unnormalized posterior distribution using a Markov chain Monte Carlo (MCMC). The algorithm used is Metropolis-Hastings [63], and instead of using multiple walkers to assure convergence, we depend on a single long chain. Ensuring that the random variable samples are independent and identically distributed (iid) and that the chain is converged to desired quantiles is done by diagnostic checks and sequential runs, following the prescriptions of Raftery and Lewis [64]. As sanity checks, all corresponding frequentist maximum likelihood estimations (MLEs) are also obtained for comparison of the best-fit results, goodness-of-fit tests, and outlier estimation. Using pulls and Cook's distances [65-67], we have ensured that none of the observables are simultaneously an outlier and a point with disproportionately large influence in our analysis. 
TABLE II. These are the 18 dimension-six effective operators (Warsaw basis) and offer additional contributions to the EWPO and Higgs signal strengths. Here, $\tau^{I}$ are normalized Pauli matrices; $I=1,2,3$.

\begin{tabular}{|c|c|c|c|c|c|}
\hline$Q_{H}$ & $\left(H^{\dagger} H\right)^{3}$ & $Q_{H G}$ & $\left(H^{\dagger} H\right) G_{\mu \nu}^{a} G^{a, \mu \nu}$ & $Q_{H e}$ & $\left(H^{\dagger} i \stackrel{\leftrightarrow}{\mathcal{D}}_{\mu} H\right)\left(\bar{e} \gamma^{\mu} e\right)$ \\
\hline$Q_{H \square}$ & $\left(H^{\dagger} H\right) \square\left(H^{\dagger} H\right)$ & $Q_{H l}^{(1)}$ & $\left(H^{\dagger} i \stackrel{\leftrightarrow}{\mathcal{D}}_{\mu} H\right)\left(\bar{l}^{\mu} l\right)$ & $Q_{H u}$ & $\left(H^{\dagger} i \stackrel{\leftrightarrow}{\mathcal{D}}_{\mu} H i\right)\left(\bar{u} \gamma^{\mu} u\right)$ \\
\hline$Q_{H D}$ & $\left(H^{\dagger} \mathcal{D}_{\mu} H\right)^{*}\left(H^{\dagger} \mathcal{D}^{\mu} H\right)$ & $Q_{H l}^{(3)}$ & $\left(H^{\dagger} i \tau^{I} \stackrel{\leftrightarrow}{\mathcal{D}}_{\mu} H\right)\left(\bar{l} \tau^{I} \gamma^{\mu} l\right)$ & $Q_{H d}$ & $\left(H^{\dagger} i \stackrel{\leftrightarrow}{\mathcal{D}}_{\mu} H\right)\left(\bar{d} \gamma^{\mu} d\right)$ \\
\hline$Q_{H B}$ & $\left(H^{\dagger} H\right) B_{\mu \nu} B^{\mu \nu}$ & $Q_{H q}^{(1)}$ & $\left(H^{\dagger} i \stackrel{\mathcal{D}}{\mathcal{D}}_{\mu} H\right)\left(\bar{q} \gamma^{\mu} q\right)$ & $Q_{e H}$ & $\left(H^{\dagger} H\right)(\bar{l} \mathrm{e} H)+$ H.c. \\
\hline$Q_{H W}$ & $\left(H^{\dagger} H\right) W_{\mu \nu}{ }^{I} W^{I, \mu \nu}$ & $Q_{H q}^{(3)}$ & $\left(H^{\dagger} i \tau^{I} \stackrel{\leftrightarrow}{\mathcal{D}}_{\mu} H\right)\left(\bar{q} \tau^{I} \gamma^{\mu} q\right)$ & $Q_{u H}$ & $\left(H^{\dagger} H\right)(\bar{q} u \tilde{H})+$ H.c. \\
\hline$Q_{H W B}$ & $\left(H^{\dagger} \tau^{I} H\right) W_{\mu \nu}^{I} B^{\mu \nu}$ & $Q_{l l}$ & $\left(\bar{l}_{\mu} l\right)\left(\bar{l} \gamma^{\mu} l\right)$ & $Q_{d H}$ & $\left(H^{\dagger} H\right)(\bar{q} d H)+$ H.c. \\
\hline
\end{tabular}

The MLE and parameter-uncertainty estimation using Hessians enables us to quickly select the initial points and proposal spreads of the MCMC chains as well.

For the first part of our analysis, we perform a model-independent statistical inference from a total of 88 observables mentioned in Sec. II in terms of 18 SMEFT dimension-six WCs as free parameters and five SM parameters $m_{Z}, m_{H}, m_{t}, \alpha_{s}\left(m_{Z}^{2}\right)$, and $\Delta \alpha_{\text {had }}^{(5)}\left(m_{Z}^{2}\right)$ as nuisance parameters. To obtain the nuisance priors, we have performed a SM fit of the EWPO, the details of which are given in Appendix A 1. The priors for the SM parameters are introduced as a multinormal distribution, following the result of the fit mentioned above. For the SMEFT model-independent analysis, uniform, uninformative priors are taken for all free parameters (WCs). We have found that the range $\{-10,10\}$ is good enough for all WCs except $\mathcal{C}_{H W}$ and $\mathcal{C}_{u H}$, for which the range $\{-50,50\}$ is chosen.

We perform two types of fits at this stage: taking all relevant WCs together, and taking one $\mathrm{WC}$ at a time. It is expected that the inferred parameter space of any one WC will be smaller for the fit with only that WC, whereas, in the presence of all other WCs, it may have a considerably larger parameter space. The results of these fits are tabulated for comparison in Table III. The first column lists the WCs, the second column is the result of the fit with all 18 WCs taken together, and in the third column, the values in each row show the result of the fit with only the corresponding WC. The fourth column lists the fit results of the ten WCs, which are generated collectively by our 11 adopted BSM scenarios. The purpose of taking these ten WCs is explained at the end of Sec. IVA. All fits are done with the cutoff set as $\Lambda=1 \mathrm{TeV}$. The correlations of the 18 WCs and ten WCs fits are tabulated in Tables XIV and XV respectively in Appendix B.

A standard analysis comparable to ours can be found in Ref. [34], and we compare the fit results mentioned in the second and third columns of Table III with those of that paper. The confidence intervals are consistent for most of the WCs, and in the following we clarify the differences. Significant dissimilarities in the parameter spaces arise due to the different observable sets considered. Unlike Ref. [34], we do not consider the differential LHC data for diboson (WW and WZ) and the Higgstrahlung (WH and $\mathrm{ZH})$ at next-to-leading-order QCD, and LEP-2 diboson data. On the other hand, we consider $m_{t}, m_{H}$, and $m_{Z}$ as observables (with dependence on WCs) in our analysis, whereas Ref. [34] considers them as auxiliary inputs. The masses being very precise put stringent constraints on the WCs that appear in their expressions. $\mathcal{C}_{H D}$ appears in the SMEFT corrections to $m_{Z}$ and $m_{H}$ and is thus more constrained in our analysis. The same is true for $\mathcal{C}_{H W B}$, $\mathcal{C}_{H \square}$, and $\mathcal{C}_{u H}$, which appear in $m_{Z}, m_{H}$, and $m_{t}$, respectively. Other than these differences, we have considered the corrections to the Higgs production and decay rates due to SMEFT operators from Ref. [62], where the kinematic differences between the production and decay channels and impact of the different center-of-mass energies on the production cross sections are neglected.

\section{MODEL-DEPENDENT ANALYSIS}

\section{A. Realizing BSMs in terms of SMEFT operators}

We consider the BSM scenarios which are single-heavymultiplet extensions of the SM. Once the massive particle (s) are integrated out, their impacts are captured through the higher-dimensional effective operators made up of SM d.o.f. Then the renormalizable BSM theories can be realized as effective ones and are expressed as

$$
\mathcal{L}_{\text {eff }}=\mathcal{L}_{\mathrm{SM}}^{d \leq 4}+\mathcal{L}_{\mathrm{SM}}^{\mathrm{EFT}}
$$

Here, $\mathcal{L}_{\mathrm{SM}}^{\mathrm{EFT}}$ can be expressed in a compact form as $\sum_{d=5, \ldots} \sum_{i}\left(\mathcal{C}_{i}^{(d)} / \Lambda^{d-4}\right) Q_{i}^{(d)}$, where the $\mathcal{C}_{i}^{(d)}$,s and $Q_{i}^{(d)}$ 's are the WCs and the SM-invariant effective operators of mass dimension $d$, respectively. Here, $i$ runs over the number of independent effective operators, i.e., the dimension of the operator basis for a given mass dimension. In this work, we restrict our study to dimension-six effective operators in the Warsaw basis only. The SM field convention is in Table IV. The SM Lagrangian is defined as 


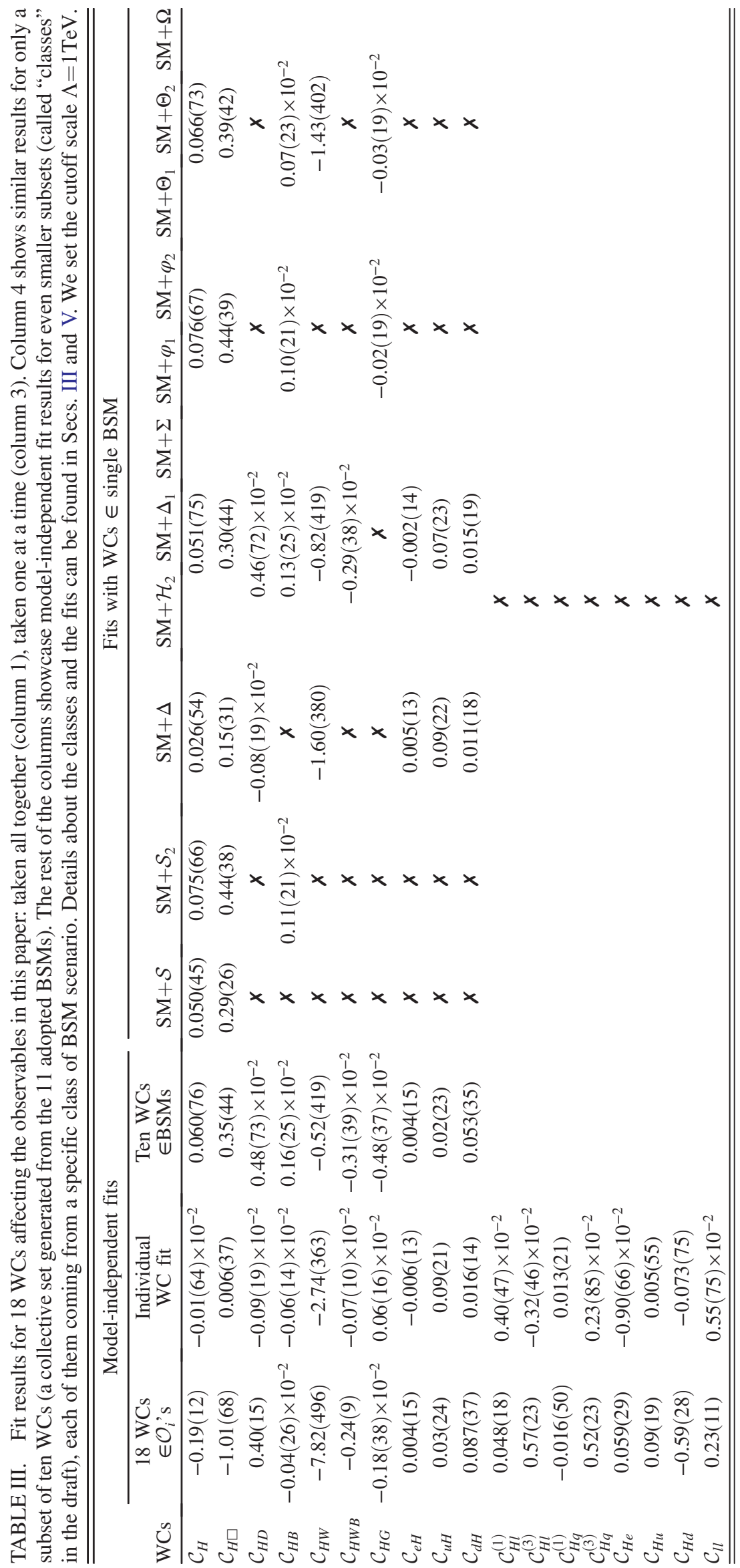




$$
\begin{aligned}
\mathcal{L}_{\mathrm{SM}}^{d \leq 4}= & -\frac{1}{4} G_{\mu \nu}^{a} G^{a, \mu \nu}-\frac{1}{4} W_{\mu \nu}^{I} W^{I, \mu \nu}-\frac{1}{4} B_{\mu \nu} B^{\mu \nu}+\left|D_{\mu} H\right|^{2}-\mu_{H}^{2}|H|^{2}-\lambda_{H}|H|^{4} \\
& +\bar{l}_{L} i \not l_{L}+\bar{q}_{L} i \emptyset q_{L}+\bar{e}_{R} i \not e_{R}+\bar{u}_{R} i \emptyset u_{R}+\bar{d}_{R} i \not d_{R} \\
& -\left\{Y_{\mathrm{SM}}^{(e)} H^{\dagger} \bar{e}_{R} l_{L}+Y_{\mathrm{SM}}^{(u)} \tilde{H}^{\dagger} \bar{u}_{R} q_{L}+Y_{\mathrm{SM}}^{(d)} H^{\dagger} \bar{d}_{R} q_{L}+\text { H.c. }\right\},
\end{aligned}
$$

where $B_{\mu \nu}, W_{\mu \nu}^{I}$, and $G_{\mu \nu}^{a}$ are the field strength tensors of the SM gauge groups $U(1)_{Y}, S U(2)_{L}$, and $S U(3)_{C}$, respectively, with $a=1, \ldots, 8$. The $D$ 's are the covariant derivatives, the $Y_{\mathrm{SM}}$ 's are the SM Yukawa couplings, and $\tilde{H}=i \sigma_{2} H^{*}$.

In the present analysis, we consider $11 \mathrm{BSM}$ scenarios which are single heavy field extensions of the SM. We choose these models carefully to encompass the variety of phenomenological features. To proceed further, we integrate out the heavy fields belonging to the adopted BSMs and compute the effective operators and their respective WCs. It is important to note here, that the WCs are the functions of BSM parameters and are thus not entirely independent. ${ }^{3}$ Here, we present the analytical structures of the WCs which are computed up to one-loop level, considering only heavy field propagators in the loop (pure heavy loop ${ }^{4}$ ) using the Mathematica package CODEx [37]. We summarize the characteristics of the to-be integrated out BSM fields corresponding to our adopted scenarios and encapsulate the respective effective operators in Table V.

In principle, though one must write down the most general gauge-invariant theories involving these heavy fields and integrate them out to compute all possible effective operators, our present analysis is driven by a set of chosen observables that encompass a very specific set of effective operators. Any other operator that does not belong to that set remains unconstrained in our analysis and is irrelevant for the purpose of this work. This is why we only note down those interactions involving the BSM fields, which can lead to the desired operators. As an example, Yukawa-type interaction terms are viable in the case of certain BSM scenarios (with the scalar as the only heavy field), and these terms generate WCs of fourfermionic effective operators that do not contribute to our set of observables. These types of interactions are ignored here, without any loss of generality. To check the complete list of WCs and their expressions in terms of BSM parameters, please go to the GitHub repository [39], where we make the full workflow and results (BSM theory implementation and the resulting effective operators and their WCs) available in Mathematica notebook files. In this work, we have ignored the renormalization group (RG)

\footnotetext{
${ }^{3}$ It is worth mentioning that this is very much unlike the usual SMEFT approach where all the WCs are assumed to be independent and free parameters.

${ }^{4}$ We have ignored the contributions from heavy-light mixing in the loop.
}

evolution of the effective operators, which are computed up to one-loop level. ${ }^{5}$ Thus, the WCs computed at the matching scale are assumed to be unaltered at the electroweak scale.

Considering the points made above, we further note that out of the 18 relevant WCs for the present set of observables, a maximum of ten operators can be exhausted (Table V) in the presence of one or more BSM scenarios of the 11 considered here. This justifies the absence of $Q_{H l}^{(1)}$, $Q_{H l}^{(3)}, Q_{H q}^{(1)}, Q_{H q}^{(3)}, Q_{H e}, Q_{H u}, Q_{H d}$, and $Q_{l l}$ operators in the model-dependent part of our analysis.

In the following subsections, we first introduce the relevant part of the BSM interactions and then tabulate the effective operators and respective WCs as functions of BSM parameters. Here, we consider the mass of the heavy fields to be the same as the cutoff scale. Thus, all the dimension-six operators are suppressed by mass-squared terms of the integrated out heavy fields.

\section{B. Real singlet scalar}

This is the first of three BSM scenarios with a unique WCs space. The SM is extended by a real singlet heavy scalar $(\mathcal{S})$ and the relevant part of interactions involving $\mathcal{S}$ is given as ${ }^{6}[28,68-70]$

$$
\begin{aligned}
\mathcal{L}_{\mathcal{S}} \supset & \frac{1}{2} D_{\mu} \mathcal{S} D^{\mu} \mathcal{S}-\frac{1}{2} m_{\mathcal{S}}^{2} \mathcal{S}^{2}-c_{\mathcal{S}, a}|H|^{2} \mathcal{S}-\frac{\kappa_{\mathcal{S}}}{2}|H|^{2} \mathcal{S}^{2} \\
& -\frac{\mu_{\mathcal{S}}}{3 !} \mathcal{S}^{3}-\frac{\lambda_{\mathcal{S}}}{4 !} \mathcal{S}^{4}
\end{aligned}
$$

where $D_{\mu}$ is the covariant derivative, ${ }^{7}$ and $m_{\mathcal{S}}$ is the mass of the heavy scalar. We list the effective operators and the respective WCs generated after $\mathcal{S}$ is integrated out in Table VI. Note that the WCs are functions of the BSM parameters depicted in Eq. (4.3). The tree-level generated WCs agree with that shown in Refs. $[32,68]$. We tabulate one-loop order WCs for pure heavy-loop processes only.

\footnotetext{
${ }^{5}$ As we have checked for some of the cases, the incorporation of RG evolution of the operators from the NP to electroweak scale does not alter our conclusion significantly and hence, is of less practical relevance.

${ }^{6}$ As the SM Lagrangian is always there for all BSMs, we are not quoting that part of the Lagrangian explicitly for each model.

${ }^{7}$ In this case, $D_{\mu}=\partial_{\mu}$ for a real singlet scalar, but for the rest of the scenarios, $D_{\mu}$ possesses nontrivial structures. As its explicit form is not required for this discussion, we will not mention it further in detail.
} 
TABLE IV. The nomenclature and quantum numbers of the SM "fields" relevant for this work. The BSM Lagrangians are defined in terms of these SM and heavy fields (Table V).

\begin{tabular}{lrccc}
\hline \hline & & \multicolumn{3}{c}{ SM quantum numbers } \\
\cline { 3 - 5 } SM fields & Spin & $S U(3)_{C}$ & $S U(2)_{L}$ & $U(1)_{Y}{ }^{a}$ \\
\hline$q_{L}$ & $\frac{1}{2}$ & 3 & 2 & $\frac{1}{6}$ \\
$l_{L}$ & $\frac{1}{2}$ & 1 & 2 & $-\frac{1}{2}$ \\
$u_{R}$ & $\frac{1}{2}$ & 3 & 1 & $\frac{2}{3}$ \\
$d_{R}$ & $\frac{1}{2}$ & 3 & 1 & $-\frac{1}{3}$ \\
$e_{R}$ & $\frac{1}{2}$ & 1 & 1 & -1 \\
$H$ & 0 & 1 & 2 & $\frac{1}{2}$ \\
$B_{\mu \nu}$ & 1 & 1 & 1 & 0 \\
$W_{\mu \nu}$ & 1 & 1 & 3 & 0 \\
$G_{\mu \nu}$ & 1 & 8 & 1 & 0 \\
\hline \hline
\end{tabular}

${ }^{\mathrm{a}}$ Hypercharge convention: $Q_{\mathrm{em}}=T_{3}+Y$, where $Q_{\mathrm{em}}, T_{3}$, and $\mathrm{Y}$ are electromagnetic charge, third component of isospin quantum number, and hypercharge, respectively.

\section{Complex singlet scalar}

In the next BSM scenario with a unique choice of WCs, the $\mathrm{SM}$ is extended by a complex singlet heavy scalar $\left(\mathcal{S}_{2}\right)$ with hypercharge $Y=2$. The relevant part of the interactions involving $\mathcal{S}_{2}$ is noted as [71,72]

$$
\begin{aligned}
\mathcal{L}_{\mathcal{S}_{2}} \supset & \left(D_{\mu} \mathcal{S}_{2}\right)^{\dagger}\left(D^{\mu} \mathcal{S}_{2}\right)-m_{\mathcal{S}_{2}}^{2} \mathcal{S}_{2}^{\dagger} \mathcal{S}_{2} \\
& -\frac{\eta_{\mathcal{S}_{2}}}{2}|H|^{2}\left|\mathcal{S}_{2}\right|^{2}-\lambda_{\mathcal{S}_{2}}\left|\mathcal{S}_{2}\right|^{4},
\end{aligned}
$$

where $m_{\mathcal{S}_{2}}$ is the mass of heavy scalar $\left(\mathcal{S}_{2}\right)$, which gets integrated out, leading to the effective operators and their respective WCs depicted in Table VII. The WCs are functions of BSM parameters; see Eq. (4.4).

\section{Isospin-triplet real scalar}

In the third and the final BSM scenario with a unique choice of WCs, the SM is extended with a color-singlet,
TABLE VI. The relevant effective operators (in the Warsaw basis) and the associated WCs generated once the heavy real SM singlet scalar $(\mathcal{S})$ is integrated out up to one-loop level are tabulated. The WCs are expressed as functions of BSM parameters; see Eq. (4.3).

\begin{tabular}{lc}
\hline \hline Effective operator & Wilson coefficient $(\mathrm{SM}+\mathcal{S})$ \\
\hline$Q_{H}$ & $-\frac{c_{\mathcal{S}, \mathrm{a}}^{2} \kappa_{S} \lambda_{\mathcal{S}}}{32 \pi^{2} m_{S}^{4}}+\frac{c_{\mathcal{S}, \mathrm{a}}^{2} \kappa_{S} \mu_{S}^{2}}{32 \pi^{2} m_{S}^{6}}-\frac{c_{\mathcal{S}, \mathrm{a}} \kappa_{S}^{2} \mu_{\mathcal{S}}}{64 \pi^{2} m_{S}^{4}}+\frac{c_{\mathcal{S}, \mathrm{a}}^{3} \lambda_{\mathcal{S}} \mu_{\mathcal{S}}}{48 \pi^{2} m_{S}^{6}}$ \\
& $-\frac{c_{\mathcal{S}, \mathrm{a}}^{3} \mu_{S}^{3}}{96 \pi^{2} m_{S}^{8}}+\frac{c_{\mathcal{S}, \mathrm{a}}^{3} \mu_{\mathcal{S}}}{6 m_{S}^{6}}-\frac{c_{\mathcal{S}, \mathrm{a}}^{2} \kappa_{S}}{2 m_{S}^{4}}-\frac{\kappa_{S}^{3}}{192 \pi^{2} m_{S}^{2}}$ \\
$Q_{H \square}$ & $-\frac{5 c_{\mathcal{S}, \mathrm{a}} \kappa_{S} \mu_{\mathcal{S}}}{192 \pi^{2} m_{S}^{4}}-\frac{c_{\mathcal{S}, \mathrm{a}}^{2} \lambda_{\mathcal{S}}}{32 \pi^{2} m_{S}^{4}}+\frac{11 c_{\mathcal{S}, \mathrm{a}}^{2} \mu_{S}^{2}}{384 \pi^{2} m_{S}^{6}}-\frac{c_{\mathcal{S}, \mathrm{a}}^{2}}{2 m_{S}^{4}}-\frac{\kappa_{S}^{2}}{384 \pi^{2} m_{S}^{2}}$ \\
\hline \hline
\end{tabular}

isospin-triplet heavy scalar $(\Delta)$ with hypercharge $Y=0$. We write the relevant interactions involving $\Delta$ as [23,29]

$$
\begin{aligned}
\mathcal{L}_{\Delta} \supset & \frac{1}{2}\left(D_{\mu} \Delta\right)^{I}\left(D^{\mu} \Delta\right)^{I}-\frac{1}{2} m_{\Delta}^{2} \Delta^{I} \Delta^{I}+2 \kappa_{\Delta} H^{\dagger} \tau^{I} H \Delta^{a} \\
& -\eta_{\Delta}|H|^{2} \Delta^{I} \Delta^{I}-\frac{\lambda_{\Delta}}{4}\left(\Delta^{I} \Delta^{I}\right)^{2},
\end{aligned}
$$

where $m_{\Delta}$ is the mass of $\Delta$. In Table VIII we list the effective operators and WCs generated after $\Delta$ is integrated out. The WCs are functions of the BSM parameters mentioned in Eq. (4.5).

\section{E. Color-singlet isospin-multiplet complex scalars}

Next, we discuss the class of BSM theories, which are extensions of the $\mathrm{SM}$ with $S U(3)_{C}$ singlet but isospin nonsinglet complex heavy scalar multiplets $\left(\mathcal{H}_{2}, \Delta_{1}, \Sigma\right)$.

$\mathrm{SM}+\mathcal{H}_{2}$.-

The $\mathrm{SM}$ is extended by a heavy $S U(2)_{L}$ complex doublet scalar $\left(\mathcal{H}_{2}\right)$ with hypercharge $Y=-\frac{1}{2}$ of mass $m_{\mathcal{H}_{2}}$. The relevant $Z_{2}$ invariant interactions of $\mathcal{H}_{2}$ are noted as $[23,73-76]$

TABLE V. SM gauge quantum numbers for the heavy BSM scalars are in the second column. Relevant effective operators are in columns 3-12. Ticks $(\boldsymbol{V})$ and crosses $(\boldsymbol{X})$ represent whether that operator (columns) is generated from the heavy fields (rows) or not. Six different classes with identical sets of operators are separated by triple lines.

\begin{tabular}{lccccccccccc}
\hline \hline $\begin{array}{l}\text { Heavy BSM } \\
\text { fields }\end{array}$ & $\begin{array}{l}\text { The SM gauge quantum no. } \\
\text { (color, isospin, hypercharge) }\end{array}$ & $Q_{H}$ & $Q_{H \square}$ & $Q_{H D}$ & $Q_{H B}$ & $Q_{H W}$ & $Q_{H W B}$ & $Q_{H G}$ & $Q_{e H}$ & $Q_{u H}$ & $Q_{d H}$ \\
\hline $\mathcal{S}$ & $(1,1,0)$ & $\checkmark$ & $\checkmark$ & $x$ & $x$ & $x$ & $x$ & $x$ & $x$ & $x$ & $x$ \\
$\mathcal{S}_{2}$ & $(1,1,2)$ & $\checkmark$ & $\checkmark$ & $x$ & $\checkmark$ & $x$ & $x$ & $x$ & $x$ & $x$ & $x$ \\
$\Delta$ & $(1,3,0)$ & $\checkmark$ & $\checkmark$ & $\checkmark$ & $x$ & $\checkmark$ & $x$ & $x$ & $\checkmark$ & $\checkmark$ & $\checkmark$ \\
$\mathcal{H}_{2}$ & $\left(1,2,-\frac{1}{2}\right)$ & $\checkmark$ & $\checkmark$ & $\checkmark$ & $\checkmark$ & $\checkmark$ & $\checkmark$ & $x$ & $\checkmark$ & $\checkmark$ & $\checkmark$ \\
$\Delta_{1}$ & $(1,3,1)$ & $\checkmark$ & $\checkmark$ & $\checkmark$ & $\checkmark$ & $\checkmark$ & $\checkmark$ & $x$ & $\checkmark$ & $\checkmark$ & $\checkmark$ \\
$\Sigma$ & $\left(1,4, \frac{1}{2}\right)$ & $\checkmark$ & $\checkmark$ & $\checkmark$ & $\checkmark$ & $\checkmark$ & $\checkmark$ & $x$ & $\checkmark$ & $\checkmark$ & $\checkmark$ \\
$\varphi_{1}$ & $\left(3,1,-\frac{1}{3}\right)$ & $\checkmark$ & $\checkmark$ & $x$ & $\checkmark$ & $x$ & $x$ & $\checkmark$ & $x$ & $x$ & $x$ \\
$\varphi_{2}$ & $\left(3,1,-\frac{4}{3}\right)$ & $\checkmark$ & $\checkmark$ & $x$ & $\checkmark$ & $x$ & $x$ & $\checkmark$ & $x$ & $x$ & $x$ \\
$\Theta_{1}$ & $\left(3,2, \frac{1}{6}\right)$ & $\checkmark$ & $\checkmark$ & $x$ & $\checkmark$ & $\checkmark$ & $x$ & $\checkmark$ & $x$ & $x$ & $x$ \\
$\Theta_{2}$ & $\left(3,2, \frac{7}{6}\right)$ & $\checkmark$ & $\checkmark$ & $x$ & $\checkmark$ & $\checkmark$ & $x$ & $\checkmark$ & $x$ & $x$ & $x$ \\
$\Omega$ & $\left(3,3,-\frac{1}{3}\right)$ & $\checkmark$ & $\checkmark$ & $x$ & $\checkmark$ & $\checkmark$ & $x$ & $\checkmark$ & $x$ & $x$ & $x$ \\
\hline \hline
\end{tabular}


TABLE VII. WCs (similar to Table VI) after integrating out the heavy complex SM singlet scalar $\left(\mathcal{S}_{2}\right)$; see Eq. (4.4).

\begin{tabular}{lc}
\hline \hline Effective operator & Wilson coefficient $\left(\mathrm{SM}+\mathcal{S}_{2}\right)$ \\
\hline$Q_{H}$ & $\frac{\eta_{\mathcal{S}_{2}}^{3}}{96 \pi^{2} m_{\mathcal{S}_{2}}^{2}}$ \\
$Q_{H B}$ & $-\frac{g_{Y}^{2} \eta_{\mathcal{S}_{2}}}{48 \pi^{2} m_{\mathcal{S}_{2}}^{2}}$ \\
$Q_{H \square}$ & $-\frac{\eta_{\mathcal{S}_{2}}^{2}}{192 \pi^{2} m_{\mathcal{S}_{2}}^{2}}$ \\
\hline \hline
\end{tabular}

TABLE VIII. WCs (similar to Table VI) after integrating out the heavy real triplet scalar $(\Delta)$; see Eq. (4.5).

\begin{tabular}{lc}
\hline \hline Effective operator & Wilson coefficient $(\mathrm{SM}+\Delta)$ \\
\hline$Q_{H}$ & $-\frac{5 \eta_{\Delta} \kappa_{\Delta}^{2} \lambda_{\Delta}}{8 \pi^{2} m_{\Delta}^{4}}-\frac{\eta_{\Delta} \kappa_{\Delta}^{2}}{m_{\Delta}^{4}}-\frac{\eta_{\Delta}^{3}}{8 \pi^{2} m_{\Delta}^{2}}+\frac{5 \lambda_{H} \kappa_{\Delta}^{2} \lambda_{\Delta}}{2 \pi^{2} m_{\Delta}^{4}}+\frac{4 \lambda_{H} \kappa_{\Delta}^{2}}{m_{\Delta}^{4}}$ \\
$Q_{H \square}$ & $-\frac{\eta_{\Delta}^{2}}{32 \pi^{2} m_{\Delta}^{2}}+\frac{5 \kappa_{\Delta}^{2} \lambda_{\Delta}}{8 \pi^{2} m_{\Delta}^{4}}+\frac{\kappa_{\Delta}^{2}}{m_{\Delta}^{4}}$ \\
$Q_{H D}$ & $-\frac{5 \kappa_{\Delta}^{2} \lambda_{\Delta}}{4 \pi^{2} m_{\Delta}^{4}}-\frac{2 \kappa_{\Delta}^{2}}{m_{\Delta}^{4}}$ \\
$Q_{H W}$ & $\frac{\eta_{\Delta} g_{W}^{2}}{96 \pi^{2} m_{\Delta}^{2}}$ \\
$Q_{e H}$ & $\frac{5 Y_{\mathrm{SM}}^{(e)} \kappa_{\Delta}^{2} \lambda_{\Delta}}{8 \pi^{2} m_{\Delta}^{4}}+\frac{Y_{\mathrm{SM}}^{(e)} \kappa_{\Delta}^{2}}{m_{\Delta}^{4}}$ \\
$Q_{u H}$ & $\frac{5 Y_{\mathrm{SM}}^{(u)} \kappa_{\Delta}^{2} \lambda_{\Delta}}{8 \pi^{2} m_{\Delta}^{4}}+\frac{Y_{\mathrm{SM}}^{(u)} \kappa_{\Delta}^{2}}{m_{\Delta}^{4}}$ \\
$Q_{d H}$ & $\frac{5 Y_{\mathrm{SM}}^{(d)} \kappa_{\Delta}^{2} \lambda_{\Delta}}{8 \pi^{2} m_{\Delta}^{4}}+\frac{Y_{\mathrm{SM}}^{(d)} \kappa_{\Delta}^{2}}{m_{\Delta}^{4}}$ \\
\hline \hline
\end{tabular}

$$
\begin{aligned}
\mathcal{L}_{\mathcal{H}_{2}} & \supset\left|D_{\mu} \mathcal{H}_{2}\right|^{2}-m_{\mathcal{H}_{2}}^{2}\left|\mathcal{H}_{2}\right|^{2}-\frac{\lambda_{\mathcal{H}_{2}}}{4}\left|\mathcal{H}_{2}\right|^{4}-\lambda_{\mathcal{H}_{2}, 1}|\tilde{H}|^{2}\left|\mathcal{H}_{2}\right|^{2} \\
& -\lambda_{\mathcal{H}_{2}, 2}\left|\tilde{H}^{\dagger} \mathcal{H}_{2}\right|^{2}-\lambda_{\mathcal{H}_{2}, 3}\left[\left(\tilde{H}^{\dagger} \mathcal{H}_{2}\right)^{2}+\left(\mathcal{H}_{2}^{\dagger} \tilde{H}\right)^{2}\right]
\end{aligned}
$$

In Table IX, we list the effective operators and their respective WC functions of the BSM parameters in Eq. (4.6), which are generated after $\mathcal{H}_{2}$ is integrated out.

$\mathrm{SM}+\Delta_{1}$. -

Here, we choose the heavy field to be an isospincomplex triplet scalar $\left(\Delta_{1}\right)$ with hypercharge $Y=1$ of mass $m_{\Delta_{1}}$. The interactions involving $\Delta_{1}$ which are relevant for us are given as [77]

$$
\begin{aligned}
\mathcal{L}_{\Delta_{1}} \supset & \operatorname{Tr}\left[\left(D_{\mu} \Delta_{1}\right)^{\dagger}\left(D^{\mu} \Delta_{1}\right)\right]-m_{\Delta_{1}}^{2} \operatorname{Tr}\left[\Delta_{1}^{\dagger} \Delta_{1}\right] \\
& -\left\{\mu_{\Delta_{1}}\left(H^{T} i \sigma_{2} \Delta_{1}^{\dagger} H\right)+\text { H.c. }\right\} \\
& -\lambda_{\Delta_{1}, 1}\left(H^{\dagger} H\right) \operatorname{Tr}\left(\Delta_{1}^{\dagger} \Delta_{1}\right)-\lambda_{\Delta_{1}, 2}\left[\operatorname{Tr}\left(\Delta_{1}^{\dagger} \Delta_{1}\right)\right]^{2} \\
& -\lambda_{\Delta_{1}, 3} \operatorname{Tr}\left[\left(\Delta_{1}^{\dagger} \Delta_{1}\right)^{2}\right]-\lambda_{\Delta_{1}, 4} H^{\dagger} \Delta_{1} \Delta_{1}^{\dagger} H .
\end{aligned}
$$

Once this field is integrated out, the same set of effective operators as the previous case are generated; see Table IX. However, the WCs are now functions of the BSM parameters given in Eq. (4.7).

$\mathrm{SM}+\Sigma$. -

The last BSM scenario considered in this class is the extension of the SM by an isospin-complex quartet heavy scalar $(\Sigma)$ with hypercharge $Y=\frac{1}{2}$ of mass $m_{\Sigma}$. The $Z_{2}$ invariant relevant interactions of $\Sigma$ are given as $[69,78,79]$

$$
\begin{aligned}
\mathcal{L}_{\Sigma} \supset & \left(D_{\mu} \Sigma\right)^{\dagger}\left(D^{\mu} \Sigma\right)-m_{\Sigma}^{2} \Sigma^{\dagger} \Sigma-\mu_{\Sigma}\left[\left(\Sigma^{\dagger} H\right)^{2}+\text { H.c. }\right] \\
& -\zeta_{1}\left(H^{\dagger} H\right)\left(\Sigma^{\dagger} \Sigma\right)-\zeta_{2}\left(H^{\dagger} \tau^{I} H\right)\left(\Sigma^{\dagger} T_{4}^{I} \Sigma\right) \\
& -\lambda_{\Sigma, 1}\left(\Sigma^{\dagger} \Sigma\right)^{2}-\lambda_{\Sigma, 2}\left(\Sigma^{\dagger} T_{4}^{I} \Sigma\right)^{2} .
\end{aligned}
$$

Once $\Sigma$ is integrated out, the same set of effective operators is generated, but the associated WCs have different functional dependence on the BSM parameters depicted in Eq. (4.8); see Table IX.

\section{F. Color-triplet isospin-singlet complex scalars}

Next, we will discuss the class of BSMs consisting of two scenarios, where the SM is extended by color-triplet, $S U(2)_{L}$ singlet heavy complex scalar fields $\left(\varphi_{1}\right.$ and $\left.\varphi_{2}\right)$ with different hypercharges.

$\mathrm{SM}+\varphi_{1}$. -

Our first choice in this category is the heavy color-triplet, isospin-singlet complex scalar $\left(\varphi_{1}\right)$ with hypercharge $Y=-\frac{1}{3}$ of mass $m_{\varphi_{1}}$. The interactions of our interest involving $\varphi_{1}$ are $[80,81]$

$$
\begin{aligned}
\mathcal{L}_{\varphi_{1}} \supset & \left(D_{\mu} \varphi_{1}\right)^{\dagger}\left(D^{\mu} \varphi_{1}\right)-m_{\varphi_{1}}^{2} \varphi_{1}^{\dagger} \varphi_{1} \\
& -\eta_{\varphi_{1}} H^{\dagger} H \varphi_{1}^{\dagger} \varphi_{1}-\lambda_{\varphi_{1}}\left(\varphi_{1}^{\dagger} \varphi_{1}\right)^{2} .
\end{aligned}
$$

In Table $\mathrm{X}$, we list the WCs along with the effective operators that are generated after $\varphi_{1}$ is integrated out. As expected, these WCs are functions of the BSM parameters noted in Eq. (4.9).

$\mathrm{SM}+\varphi_{2}$.-

Another scenario belonging to the same class is found when the SM is extended by a color-triplet, isospin-singlet complex scalar $\left(\varphi_{2}\right)$ of hypercharge $\mathrm{Y}=-\frac{4}{3}$ and of mass $m_{\varphi_{2}}$. The interactions of our interest involving $\varphi_{2}$ are $[82,83]$

$$
\begin{aligned}
\mathcal{L}_{\varphi_{2}} \supset & \left(D_{\mu} \varphi_{2}\right)^{\dagger}\left(D^{\mu} \varphi_{2}\right)-m_{\varphi_{2}}^{2} \varphi_{2}^{\dagger} \varphi_{2} \\
& -\eta_{\varphi_{2}} H^{\dagger} H \varphi_{2}^{\dagger} \varphi_{2}-\lambda_{\varphi_{2}}\left(\varphi_{2}^{\dagger} \varphi_{2}\right)^{2} .
\end{aligned}
$$

We find that the exact set of effective operators is generated as $\varphi_{1}$ once $\varphi_{2}$ is integrated out. For this case, the WC functions of the BSM parameters noted in Eq. (4.10) are captured in Table X.

\section{G. Color-triplet isospin-multiplet complex scalars}

The last class of BSM scenarios are extensions of the SM with heavy complex scalar fields $\Theta_{1}, \Theta_{2}$, and $\Omega$ charged under both color and isospin symmetries.

$\mathrm{SM}+\Theta_{1}$. -

The first one is the extension of the SM with a color-triplet, isospin-doublet complex scalar $\left(\Theta_{1}\right)$ with 
TABLE IX. WCs (similar to Table VI) after integrating out the heavy complex scalars $\mathcal{H}_{2}, \Delta_{1}$, and $\Sigma$; see Eqs. (4.6)-(4.8).

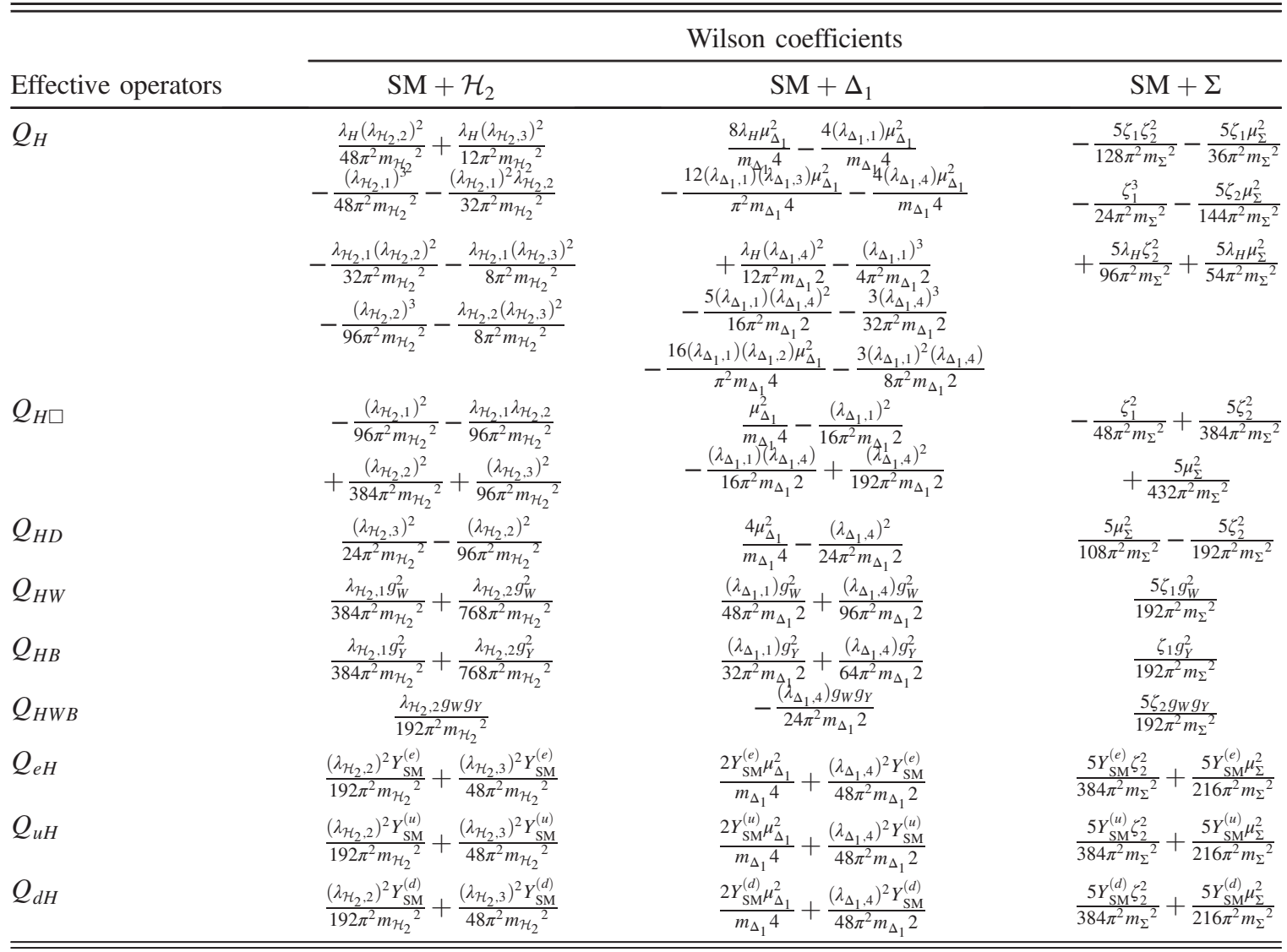

TABLE X. WCs (similar to Table VI) after integrating out the heavy scalars $\varphi_{1}$ and $\varphi_{2}$; see Eqs. (4.9) and (4.10).

\begin{tabular}{lcc}
\hline \hline & \multicolumn{2}{c}{ Wilson coefficients } \\
\cline { 2 - 3 } Effective operator & $\mathrm{SM}+\varphi_{1}$ & $\mathrm{SM}+\varphi_{2}$ \\
\hline$Q_{H}$ & $-\frac{\eta_{\varphi_{1}}^{3}}{32 \pi^{2} m_{\varphi_{1}}^{2}}$ & $-\frac{\eta_{\varphi_{2}}^{3}}{32 \pi^{2} m_{\varphi_{2}}^{2}}$ \\
$Q_{H \square}$ & $-\frac{\eta_{\varphi_{1}}}{64 \pi^{2} m_{\varphi_{1}}^{2}}$ & $-\frac{\eta_{\varphi_{2}}}{64 \pi^{2} m_{\varphi_{2}}^{2}}$ \\
$Q_{H B}$ & $\frac{g_{Y}^{2} \eta_{\varphi_{1}}}{576 \pi^{2} m_{\varphi_{1}}^{2}}$ & $\frac{g_{Y}^{2} \eta_{\varphi_{2}}}{36 \pi^{2} m_{\varphi_{2}}^{2}}$ \\
$Q_{H G}$ & $\frac{g_{S}^{2} \eta_{\varphi_{1}}}{384 \pi^{2} m_{\varphi_{1}}^{2}}$ & $\frac{g_{S}^{2} \eta_{\varphi_{2}}}{384 \pi^{2} m_{\varphi_{2}}^{2}}$ \\
\hline \hline
\end{tabular}

hypercharge $Y=\frac{1}{6}$ of mass $m_{\Theta_{1}}$. The relevant part of the interactions involving $\Theta_{1}$ is $[83,84]$

$$
\begin{aligned}
\mathcal{L}_{\Theta_{1}} \supset & \left(D_{\mu} \Theta_{1}\right)^{\dagger}\left(D^{\mu} \Theta_{1}\right)-m_{\Theta_{1}}^{2} \Theta_{1}^{\dagger} \Theta_{1} \\
& -\eta_{\Theta_{1}} H^{\dagger} H \Theta_{1}^{\dagger} \Theta_{1}-\lambda_{\Theta_{1}}\left(\Theta_{1}^{\dagger} \Theta_{1}\right)^{2} .
\end{aligned}
$$

The effective operators and the associated WCs that are generated after integrating out $\Theta_{1}$ are depicted in Table XI.
The WCs are expressed in terms of the BSM parameters mentioned in Eq. (4.11).

$\mathrm{SM}+\Theta_{2}$.-

In the second instance, the heavy field $\left(\Theta_{2}\right)$ with mass $m_{\Theta_{2}}$ is a color-triplet, isospin-doublet complex scalar with hypercharge $Y=\frac{7}{6}$. The interactions of $\Theta_{2}$ that are relevant to our analysis are [82-84]

$$
\begin{aligned}
\mathcal{L}_{\Theta_{2}} \supset & \left(D_{\mu} \Theta_{2}\right)^{\dagger}\left(D^{\mu} \Theta_{2}\right)-m_{\Theta_{2}}^{2} \Theta_{2}^{\dagger} \Theta_{2} \\
& -\eta_{\Theta_{2}} H^{\dagger} H \Theta_{2}^{\dagger} \Theta_{2}-\lambda_{\Theta_{2}}\left(\Theta_{2}^{\dagger} \Theta_{2}\right)^{2} .
\end{aligned}
$$

Similar to the previous case, the same operators are generated once $\Theta_{2}$ is integrated out. The WC functions of the BSM parameters [Eq. (4.12)] are listed in Table XI.

$\mathrm{SM}+\Omega$.

In the last example model under this class, we choose the heavy field to be a color-triplet, isospin-triplet scalar $(\Omega)$ with hypercharge $Y=-\frac{1}{3}$ and mass $m_{\Omega}$. The interactions involving $\Omega$ that are relevant for this work are $[83,84]$

$$
\mathcal{L}_{\Omega} \supset\left(D_{\mu} \Omega\right)^{\dagger}\left(D^{\mu} \Omega\right)-m_{\Omega}^{2} \Omega^{\dagger} \Omega-\eta_{\Omega} H^{\dagger} H \Omega^{\dagger} \Omega-\lambda_{\Omega}\left(\Omega^{\dagger} \Omega\right)^{2} .
$$


TABLE XI. WCs (similar to Table VI) after integrating out the heavy scalars $\Theta_{1}, \Theta_{2}$, and $\Omega$; see Eqs. (4.11)-(4.13).

\begin{tabular}{lccc}
\hline \hline & \multicolumn{3}{c}{ Wilson coefficients } \\
\cline { 2 - 4 } Effective operator & $\mathrm{SM}+\Theta_{1}$ & $\mathrm{SM}+\Theta_{2}$ & $\mathrm{SM}+\Omega$ \\
\hline$Q_{H}$ & $-\frac{\eta_{\Theta_{1}}^{3}}{16 \pi^{2} m_{\Theta_{1}}^{2}}$ & $-\frac{\eta_{\Theta_{2}}^{3}}{16 \pi^{2} m_{\Theta_{2}}^{2}}$ & $-\frac{3 \eta_{\Omega}^{3}}{32 \pi^{2} m_{\Omega}^{2}}$ \\
$Q_{H \square}$ & $-\frac{\eta_{\Theta_{1}}^{2}}{32 \pi^{2} m_{\Theta_{1}}^{2}}$ & $-\frac{\eta_{\Theta_{2}}^{2}}{32 \pi^{2} m_{\Theta_{2}}^{2}}$ & $-\frac{3 \eta_{\Omega}^{2}}{64 \pi^{2} m_{\Omega}^{2}}$ \\
$Q_{H B}$ & $\frac{g_{Y}^{2} \eta_{\Theta_{1}}}{1152 \pi^{2} m_{\Theta_{1}}^{2}}$ & $\frac{49 g_{Y}^{2} \eta_{\Theta_{2}}}{1152 \pi^{2} m_{\Theta_{2}}^{2}}$ & $\frac{g_{Y}^{2} \eta_{\Omega}}{192 \pi^{2} m_{\Omega}^{2}}$ \\
$Q_{H G}$ & $\frac{g_{W}^{2} \eta_{\Theta_{1}}}{12 \pi^{2} m_{\Theta_{1}}^{2}}$ & $\frac{g_{W}^{2} \eta_{\Theta_{2}}}{12 \pi^{2} m_{\Theta_{2}}^{2}}$ & $\frac{g_{W}^{2} \eta_{\Omega}}{32 \pi^{2} m_{\Omega}^{2}}$ \\
$Q_{H W}$ & $\frac{g_{S}^{2} \eta_{\Theta_{1}}}{192 \pi^{2} m_{\Theta_{1}}^{2}}$ & $\frac{g_{S}^{2} \eta_{\Theta_{2}}}{192 \pi^{2} m_{\Theta_{2}}^{2}}$ & $\frac{g_{S}^{2} \eta_{\Omega}}{128 \pi^{2} m_{\Omega}^{2}}$ \\
\hline \hline
\end{tabular}

Once $\Omega$ is integrated out, the same set of effective operators similar to the previous two scenarios emerge. In Table XI, we capture the WCs which are functions of the model parameters given in Eq. (4.13).

\section{H. Statistical inference}

To obtain the bounds on the model parameters, first the low-energy observables are written in terms of dimensionsix SMEFT WCs and the SM parameters and then they are mapped to the effective theory by expressing the WCs as functions of the BSM couplings and the cutoff scale $\Lambda^{8}$ following Secs. IV B-IV G.

The statistical methodology is similar to that described in Sec. III B, with the exception that the free fit parameters here are those of the BSM models. In general, we choose uniform priors of $\{-4 \pi, 4 \pi\}$ for BSM quartic couplings due to perturbativity and $\{-\Lambda, \Lambda\}$ for couplings with mass dimension one (since the cutoff scale $\Lambda=1 \mathrm{TeV}$ ). In certain scenarios, the Bayesian fit is insensitive to certain model parameters, and in most cases, these insensitive parameters are BSM self-quartic couplings.

In the following, we discuss the fits of the model parameter space of different SM extensions. We first discuss the models with multiple parameters followed by single parameter models.

\section{Models with multiple parameters}

$\mathrm{SM}+\mathcal{S}$.-

In this model, we find a set of two WCs $\mathcal{C}_{H}$ and $\mathcal{C}_{H \square}$ that depend upon the model parameters $c_{\mathcal{S}, a}, \kappa_{\mathcal{S}}, \mu_{\mathcal{S}}$, and $\lambda_{\mathcal{S}}$ (see column 2 of Table VI). We perform the fit assuming the uniform priors of $\{-\Lambda, \Lambda\}$ for $c_{\mathcal{S}, a}$ and $\mu_{\mathcal{S}}$, and $\{-4 \pi, 4 \pi\}$ for $\kappa_{\mathcal{S}}$ and $\lambda_{\mathcal{S}}$. We find that the observables used in this analysis are insensitive to $\mu_{\mathcal{S}}$ and $\lambda_{\mathcal{S}}$; i.e., these have negligible effects on the posterior distributions of the other

\footnotetext{
${ }^{8}$ The masses of the heavy scalars are equivalent to the cutoff scale $\Lambda$, which is chosen to be $1 \mathrm{TeV}$ for the entirety of the analysis.
}

parameters. Therefore, without loss of generality, these are set to be zero, and the fit results of $c_{\mathcal{S}, a}$ and $\lambda_{\mathcal{S}}$ are obtained along with five SM parameters. The one-dimensional (showing individual parameter space) and two-dimensional (showing correlations) marginal posterior distributions encapsulating the correlations between them are shown in Fig. 1(a).

$\mathrm{SM}+\Delta .-$

The seven WCs in the effective theory resulting from extending the SM with a heavy real $S U(2)_{L}$ triplet scalar $(\Delta)$ constrain three model parameters $\eta_{\Delta}, \kappa_{\Delta}$, and $\lambda_{\Delta}$ (see the second column of Table VII). The ranges $\{-4 \pi, 4 \pi\}$ for $\eta_{\Delta}$ and $\lambda_{\Delta}$, and $\{-\Lambda, \Lambda\}$ for $\kappa_{\Delta}$ are set as uniform priors. The marginal posteriors are shown in Fig. 1(b).

\section{$\mathrm{SM}+\Delta_{1}$}

For the complex $S U(2)_{L}$ triplet scalar $\left(\Delta_{1}\right)$ extension, a set of nine WCs is related to the five model parameters $\mu_{\Delta_{1}}$, $\lambda_{\Delta_{1},\{1,2,3,4\}}$ (third column of Table IX). The fit is performed taking uniform priors within the range of $\{-4 \pi, 4 \pi\}$ for the scalar quartic couplings $\lambda_{\Delta_{1},\{1,2,3,4\}}$ and $\{-\Lambda, \Lambda\}$ for $\mu_{\Delta_{1}}$ along with the SM parameters. Just like the model above, the fit is insensitive to $\lambda_{\Delta_{1}, 2}$, and $\lambda_{\Delta_{1}, 3}$, and hence, they are set to zero. The marginal posteriors of $\lambda_{\Delta_{1}, 1}, \lambda_{\Delta_{1}, 4}$, and $\mu_{\Delta_{1}}$ are shown in Fig. 1(c).

$\mathrm{SM}+\mathcal{H}_{2}$.-

We explore the model parameter space of the $\mathcal{H}_{2}$ extended BSM scenario utilizing the nine WCs written as functions of three model parameters listed in the second column of Table IX. The BSM quartic couplings $\lambda_{\mathcal{H}_{2}, 1}$, $\lambda_{\mathcal{H}_{2}, 2}$, and $\lambda_{\mathcal{H}_{2}, 3}$ are fitted along with the SM parameters assuming uniform priors of $\{-4 \pi, 4 \pi\}$. The one- and twodimensional marginal posterior for the BSM parameters $\lambda_{\mathcal{H}_{2}, 1}, \lambda_{\mathcal{H}_{2}, 2}$, and $\lambda_{\mathcal{H}_{2}, 3}$ are presented in the Fig. 1(d).

$\mathrm{SM}+\Sigma$. -

An analysis similar to those described above for different BSM extensions is done for the model parameters of $\mathrm{SM}+\Sigma$ using the one-loop matching results. In this $S U(2)_{L}$ quartet scalar extension of $\Sigma$, a list of nine WCs are expressed as functions of three model parameters $\zeta_{1}, \zeta_{2}$, and $\mu_{\Sigma}$ (see the fourth column of Table IX). The posterior distributions of these model parameters, along with SM parameters, are sampled assuming uniform priors of $\{-4 \pi, 4 \pi\}$ for $\zeta_{1}, \zeta_{2}$, and $\mu_{\Sigma}$. The one-dimensional marginal posteriors of $\zeta_{1}, \zeta_{2}$, and $\mu_{\Sigma}$, along with their two-dimensional counterparts, are shown in Fig. 1(e).

\section{Models with one parameter}

In the rest of the BSM scenarios containing heavy scalars $\mathcal{S}_{2}, \varphi_{1}, \varphi_{2}, \Omega, \Theta_{1}, \Theta_{2}$, the WCs are functions of only one BSM parameter for each model. The fit results are shown in Table XII. We refrain from showing their posteriors, as these are quite imprecise with a large, equiprobable parameter space while being consistent with zero. 


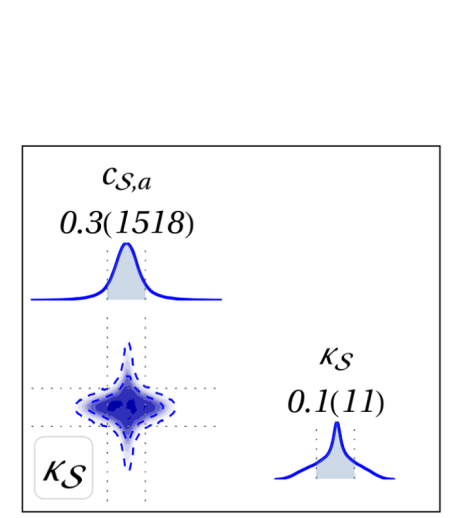

(a) $\mathrm{SM}+\mathcal{S}$

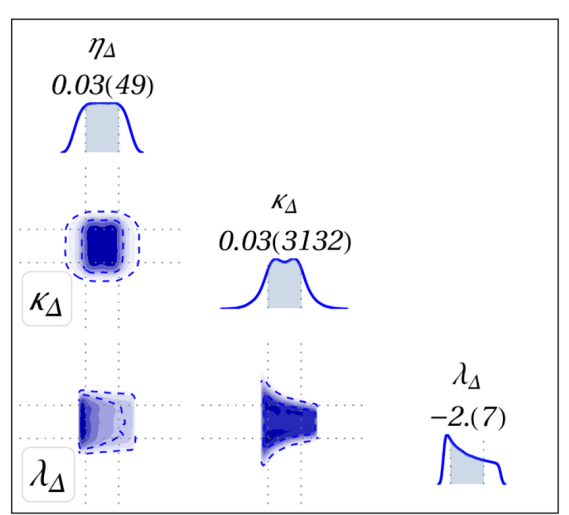

(b) $\mathrm{SM}+\Delta$

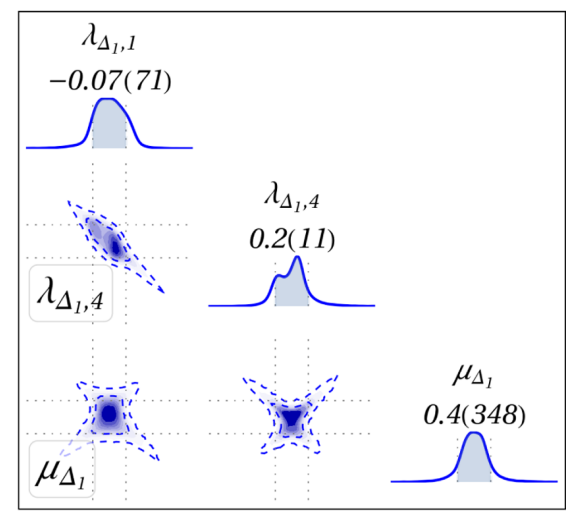

(c) $\mathrm{SM}+\Delta_{1}$

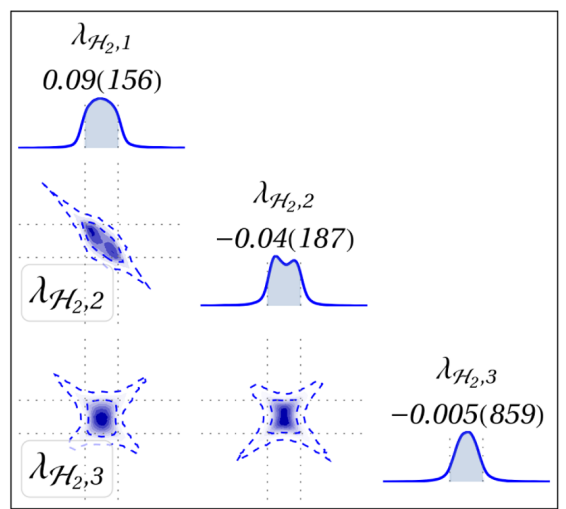

(d) $\mathrm{SM}+\mathcal{H}_{2}$

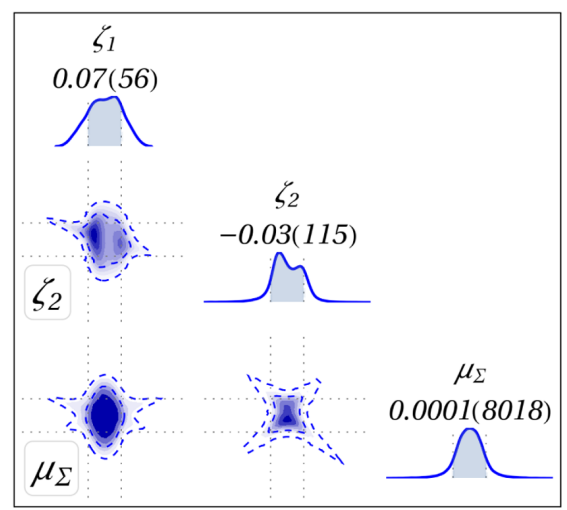

(e) $\mathrm{SM}+\Sigma$

FIG. 1. The one- and two-dimensional marginalized posteriors for different $\mathrm{SM}$ extensions (a) $\mathrm{SM}+\mathcal{S}$, (b) $\mathrm{SM}+\Delta$, (c) $\mathrm{SM}+\Delta_{1}$, (d) $\mathrm{SM}+\mathcal{H}_{2}$, and (e) $\mathrm{SM}+\sum$ showing the correlations among them. Only the BSM parameters are shown. For full tri-plots like these, including the SM-parameters, please check our GitHub repository [39]. Here, the fitted values of $c_{\mathcal{S}, a}, \kappa_{\Delta}$, and $\mu_{\Delta_{1}}$ are expressed in GeV.

\section{MODEL-INDEPENDENT VS MODEL- DEPENDENT ANALYSES}

Comparing the span of the WCs for the fit with all effective operators present and fits with one $\mathrm{WC}$ at a time (second and third columns of Table III) shows us how the allowed space of each WC increases in the presence of other independent WCs. This non-negligible variation of the WC space is the reason for our including the ten WCs fit result in the fourth column of the said table. This enunciates the fact that for a meaningful comparison of parameter spaces between model-independent and model-dependent studies, we need to consider only those subsets of the WCs which come from some BSM model considered here. As can be seen from Table V, some of the BSM scenarios considered in this analysis give rise to the same set of effective operators. This observation motivates us to categorize these 11 scenarios into six different classes of BSM theories.

Our rule of thumb is if two or more BSM scenarios lead to the same set of effective operators, they are declared degenerate and are categorized into a single class. It is important to remark that though the degenerate models possess the same operators, their respective WCs, which depend on model parameters, are entirely different, reflecting the intrinsic nonidentical nature of those BSMs. This strategy of categorizing multiple models together in a class, using the power of EFT, enables us to bring down some of the BSMs in the same footing to be adjudged simultaneously, and thus to allow further comparative remarks. This methodology is illustrated by the flowchart in Fig. 2.

TABLE XII. Bayesian fit results of BSM theories with a single parameter.

\begin{tabular}{lcccccc}
\hline \hline Model & $\mathrm{SM}+\mathcal{S}_{2}$ & $\mathrm{SM}+\varphi_{1}$ & $\mathrm{SM}+\varphi_{2}$ & $\mathrm{SM}+\Omega$ & $\mathrm{SM}+\Theta_{1}$ & $\mathrm{SM}+\Theta_{2}$ \\
\hline Model & $\eta_{\mathcal{S}_{2}}=$ & $\eta_{\varphi_{1}}=$ & $\eta_{\varphi_{2}}=$ & $\eta_{\Omega}=$ & $\eta_{\Theta_{1}}=$ & $\eta_{\Theta_{2}}=$ \\
Parameter & $0.067 \pm 1.141$ & $0.076 \pm 0.794$ & $0.016 \pm 0.793$ & $0.093 \pm 0.533$ & $0.095 \pm 0.619$ & $0.032 \pm 0.622$ \\
\hline \hline
\end{tabular}




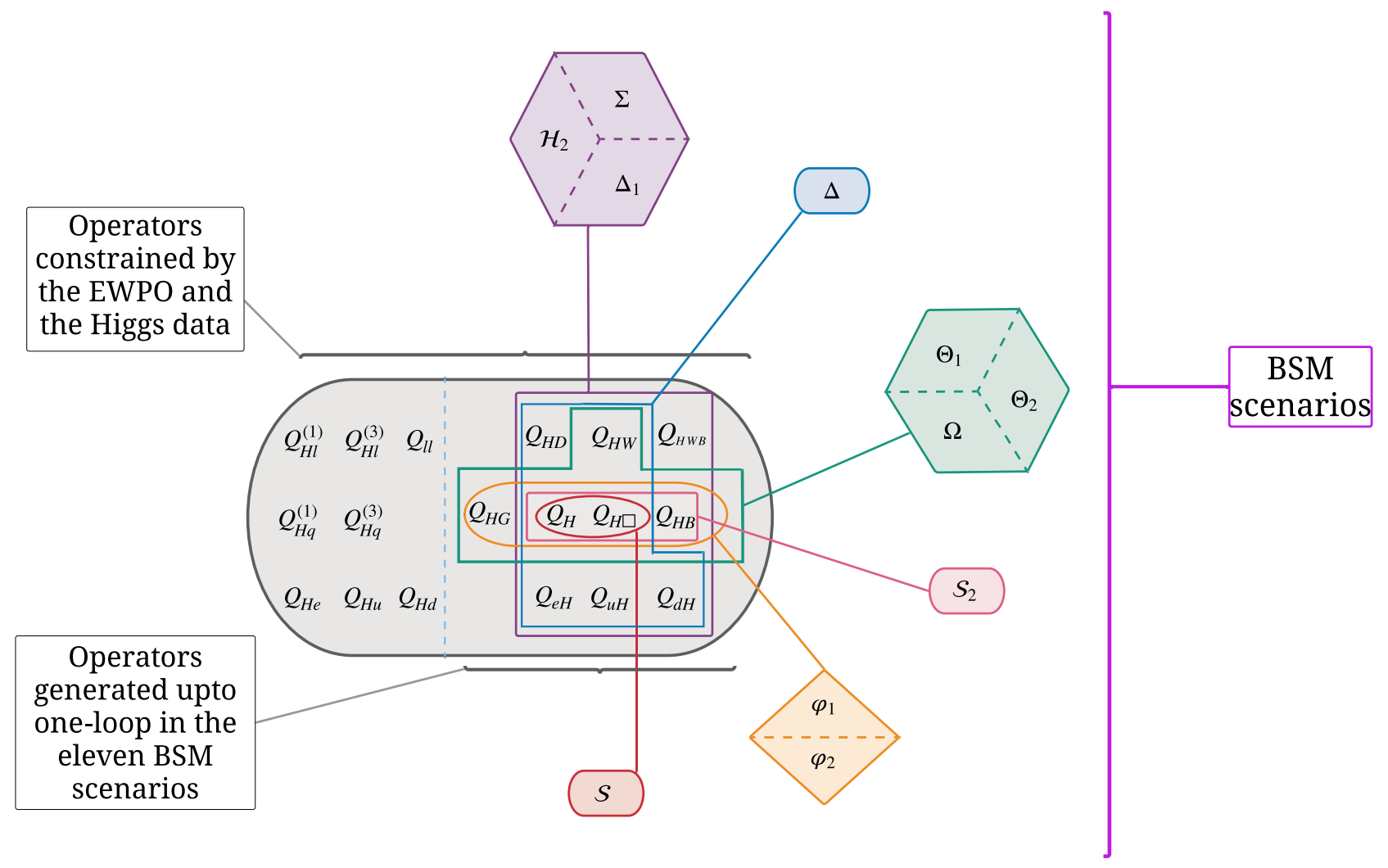

FIG. 2. This flowchart encapsulates the "model-independent" vs "model-dependent" analysis discussed in Sec. V.

For each of these six classes, we first want to constrain the WCs from the data in a model-independent manner. To this end, we obtain the Bayesian inference on these classes of WCs without any model information and by varying them as free and independent parameters in each case. The operators that are not generated in a given model are taken to be zero. These results from now on will be considered to be the "model-independent" results. The remaining columns of Table III list the best-fit values and uncertainties for each class. As can be seen from the second row (from top) in that table, the last three columns are the results for classes with WCs, which can be connected to multiple BSM scenarios. The results clearly show the necessity of considering these individual classes, as the parameter space of each WC varies largely from both the fit with all WCs present and the fit with only that WC, as well as fits for other classes. As an example, one can follow the row of $\mathcal{C}_{H \square}$ in Table III and see how even the model-independent results vary.

In the next step, using the data-driven BSM parameter posteriors of each model of a given class (Sec. IV H) and the matching the results relating the WCs to the BSM parameters obtained using CoDEx (Tables VII-IX), we proceed to constrain the same set of WCs again. In this case, we expect to obtain a different result for each BSM model, as the WCs are functions of the BSM parameters and thus related to each other. We use the large samples generated in the MCMC processes for each model fit to obtain the multivariate distributions of corresponding WCs. Where the model-independent results for each class do not contain any model information, the WC distributions generated in this way are naturally highly constrained by the structure of the specific BSM models. Comparing the WC distributions obtained in these two ways is effectively one definitive way of comparing the model-independent and -dependent results.

To visualize these distributions, we use marginal posteriors for the WC distributions taken two at a time. For a specific class, the model-independent two-dimensional marginal posterior is the same, while those generated directly from several BSM scenarios from the same class are different. Whenever possible, we compare them together.

Figure 3 showcases these results for the class of models $\mathrm{SM}+\varphi_{1}$ and $\mathrm{SM}+\varphi_{2}$ with four associated effective SMEFT operators. The large gray regions correspond to the model-independent $68 \%$ and $95 \%$ credible intervals (darker to lighter) of corresponding WCs. Similarly, the red-dashed-bounded and blue regions correspond to the $\mathrm{WC}$ regions obtained from models $\mathrm{SM}+\varphi_{1}$ and $\mathrm{SM}+\varphi_{2}$, respectively. As the latter ones occupy comparatively tiny regions in the main figure, we show their enlarged versions in the inset. As we are mainly interested in the allowed parameter spaces for individual WCs from modeldependent or -independent analyses, we show only the 


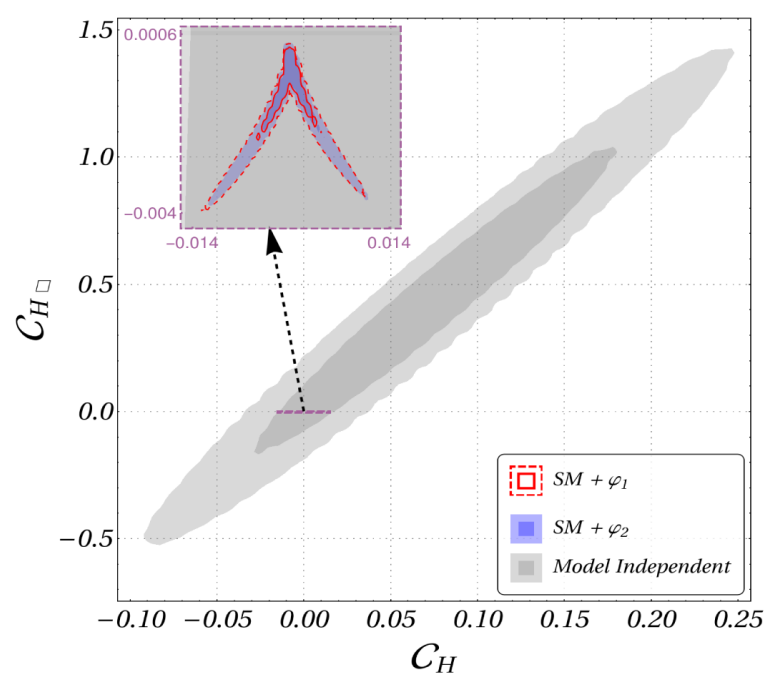

(a) $\mathcal{C}_{H}-\mathcal{C}_{H \square}$

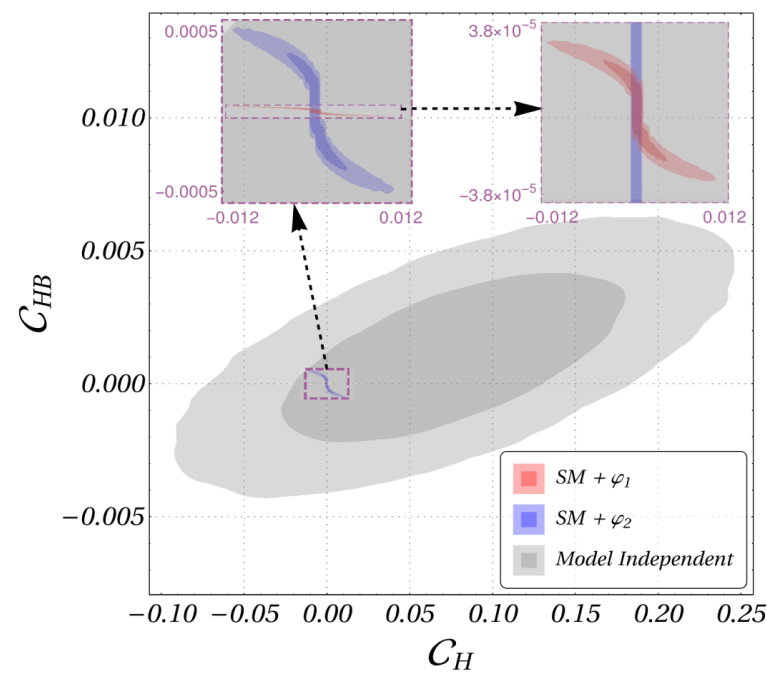

(b) $\mathcal{C}_{H}-\mathcal{C}_{H B}$

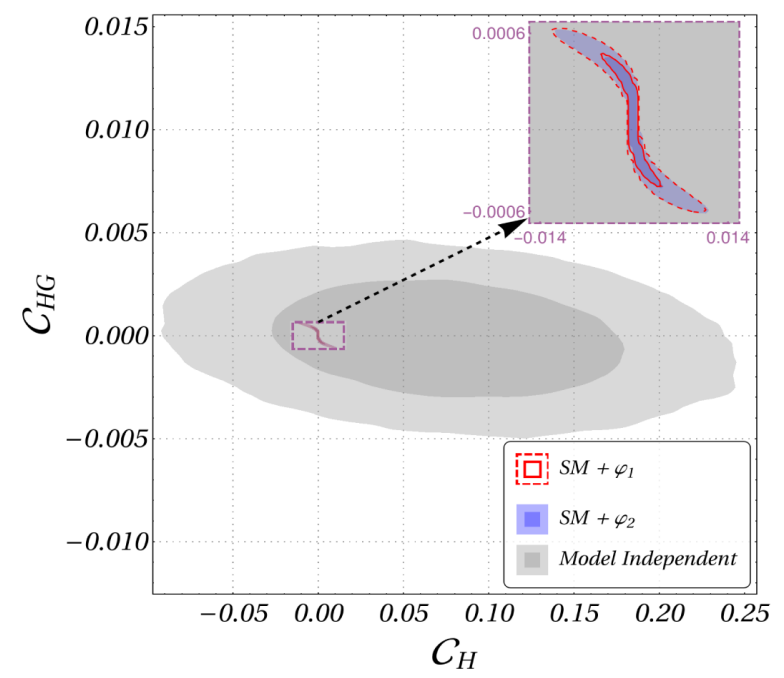

(c) $\mathcal{C}_{H}-\mathcal{C}_{H G}$

FIG. 3. Comparison of the two-dimensional posteriors of model-independent WCs (gray; 68\% (darker) and 95\% (lighter) credible intervals) with those generated from the class of degenerate leptoquark singlet scenarios. The red-dashed-bounded and blue regions correspond to the similar regions obtained from the $\mathrm{SM}+\varphi_{1}$ and $\mathrm{SM}+\varphi_{2}$ respectively. Enlarged spaces are shown in the inset. Figs. 3 (a)-3(c) show the two-dimensional posteriors for $C_{H}-C_{H \square}, C_{H}-C_{H B}$, and $C_{H}-C_{H G}$ respectively.

smallest subset of possible two-dimensional marginal distributions here onward containing all the WC regions. These, among all other possible figures not shown here, are organized in the GitHub repository associated with this work [39].

For the sake of clarity, let us discuss Fig. 3 in detail here. We have seen from Sec. IV F that the two BSM models with extra color-triplet, isospin-singlet complex scalars with different hypercharges give rise to the same set of four effective operators $\left(Q_{H}, Q_{H \square}, Q_{H B}\right.$, and $\left.Q_{H G}\right)$ relevant to our observables. Thus, we put them in the same class of models following the prescription at the beginning of this section. Each model in this class has only one BSM parameter, whose parameter spaces are quoted in Table XII.
From a model-independent point of view, if we just keep the four WCs $\left(C_{H}, C_{H \square}, C_{H B}\right.$, and $\left.C_{H G}\right)$ as independent parameters in the fit and vary them simultaneously, the obtained parameter space is the one we get in the penultimate column of Table III. This, in our result, constitutes a four-dimensional posterior in the WC space. If we marginalize to just two WCs $C_{H}$ and $C_{H \square}$ (not by setting the other WCs to their best-fit value, but rather taking a twodimensional projection of the whole posterior), we get the large gray elliptical shape in Fig. 3(a), the darker (lighter) region of which is the 2D 68\% (95\%) credible region. The tilt of the ellipse shows a positive correlation between the WCs, which can be found to be 0.995 . To show the optimal amount of information in a minimum number of figures, we 


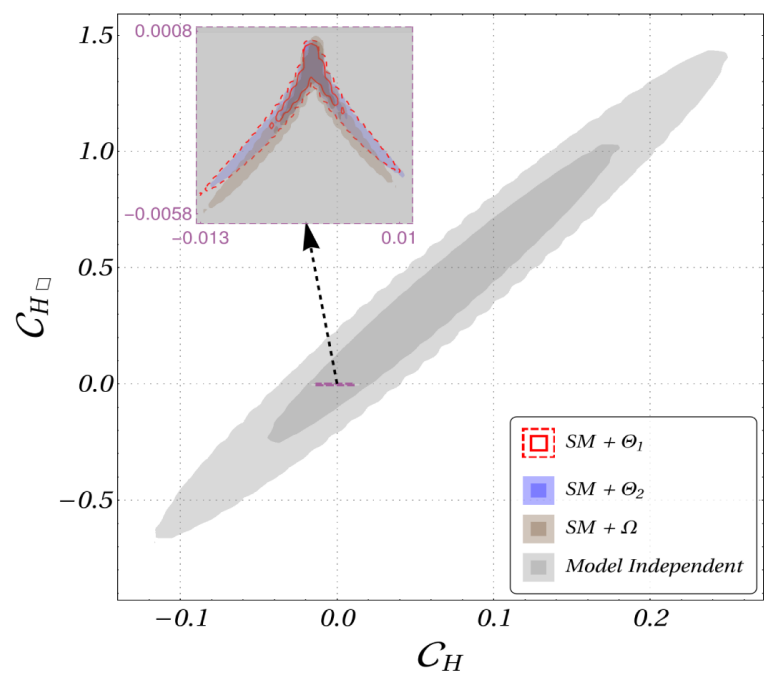

(a) $\mathcal{C}_{H}-\mathcal{C}_{H \square}$

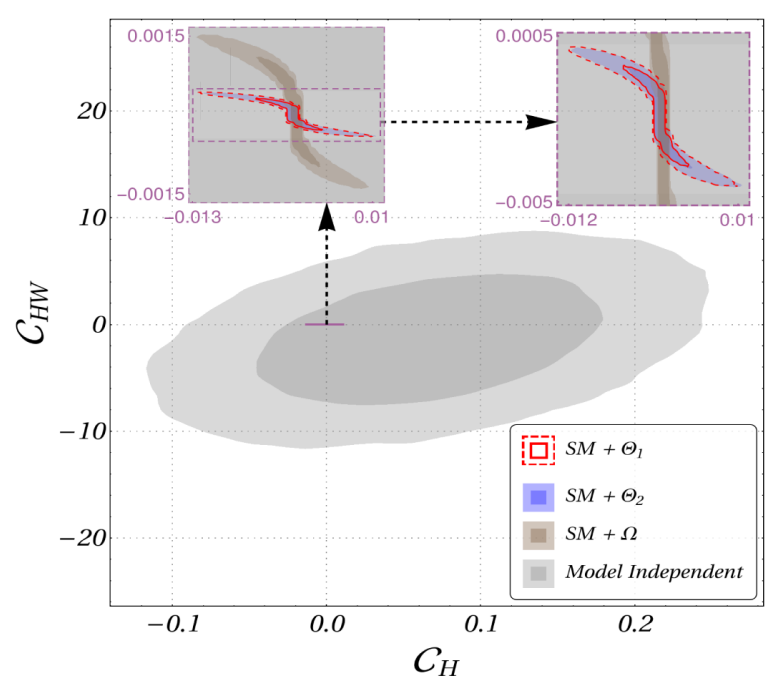

(c) $\mathcal{C}_{H}-\mathcal{C}_{H W}$

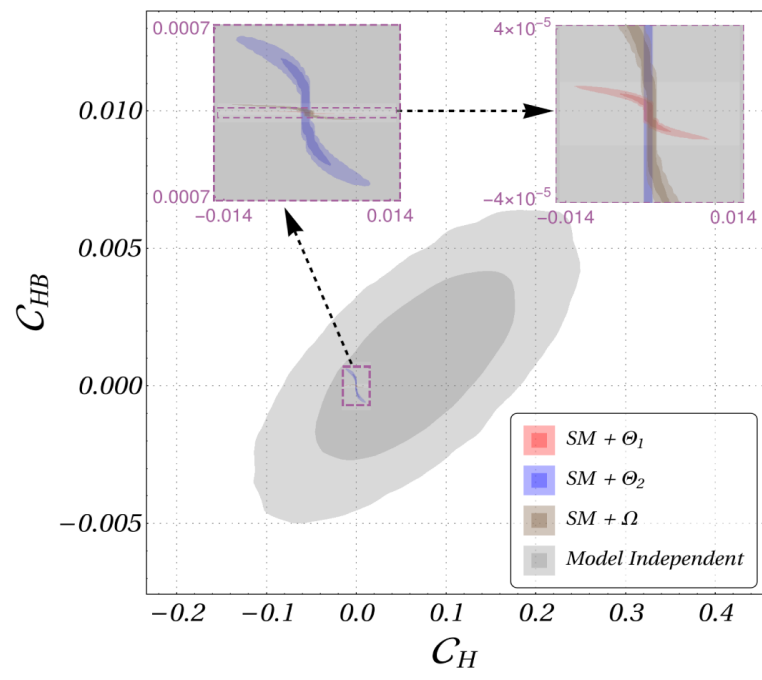

(b) $\mathcal{C}_{H}-\mathcal{C}_{H B}$

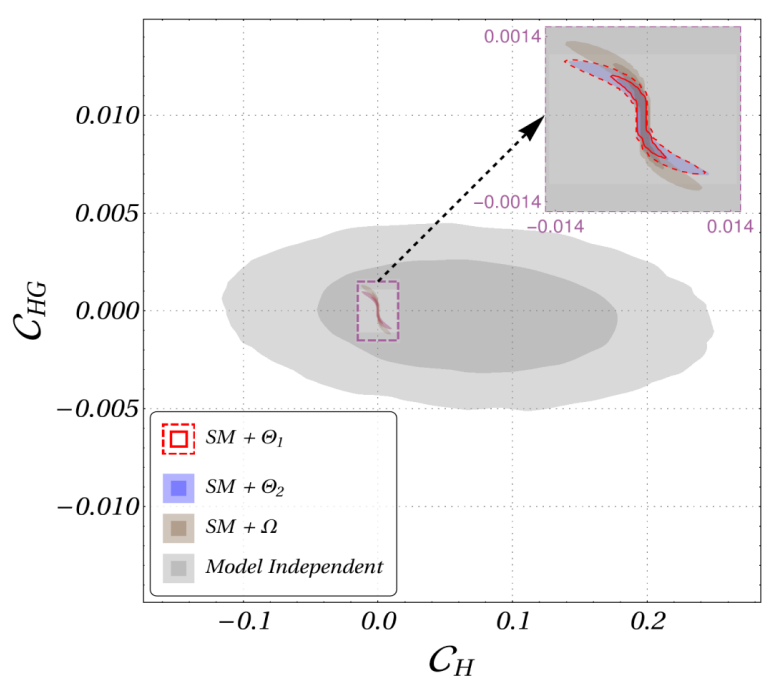

(d) $\mathcal{C}_{H}-\mathcal{C}_{H G}$

FIG. 4. Figs. 4(a)-4(d) similar to Fig. 3 corresponding to the class of leptoquark-multiplet scenarios, namely, $\mathrm{SM}+\Theta_{1}, \mathrm{SM}+\Theta_{2}$, and $\mathrm{SM}+\Omega$.

have kept the $x$ axis of the other two plots of Fig. 3 fixed to $C_{H}$ and varied the $y$ axis. To compare this model-independent parameter space with individual models, we then have propagated the posteriors of the individual model parameters and found the WC space for them as well. For example, the model-dependent WC space for the BSM model SM $+\varphi_{2}$ is the blue region in all plots of Fig. 3. As can be seen, these WC spaces are found to be orders of magnitude smaller than the model-independent ones, and hence, we have enlarged them in the inset. Sometimes, one round of magnification is not enough, as is evident from Fig. 3(b), which shows that the WC space corresponding to $\mathrm{SM}+\varphi_{1}$ is orders of magnitude smaller than even that of $\mathrm{SM}+\varphi_{2}$, thus necessitating two rounds of magnification in the second inset.

Similar figures are obtained for the rest of the classes with multiple models. The WC spaces for models
$\mathrm{SM}+\Theta_{1}, \mathrm{SM}+\Theta_{2}$, and $\mathrm{SM}+\Omega$ with five effective SMEFT operators are shown in Fig. 4, whereas Figs. 5 and 6 show those regions for the class of models involving $S U(2)_{L}$ doublet, triplet, and quartet scalars with nine mapped SMEFT operators.

The WC spaces for classes with a single model in each, namely, $\mathrm{SM}+\Delta, \mathrm{SM}+\mathcal{S}$, and $\mathrm{SM}+\mathcal{S}_{2}$ are shown in Figs. 7 and 8 , respectively. There is a single connecting theme throughout all these figures: the minuscule size of the model-constrained WC regions compared to their modelindependent counterparts. Though all model-independent WC regions are consistent with the SM, looking only at the model-independent WC spaces, one erroneously concludes that any model giving rise to these WCs would probably have quite a large parameter space allowed, whereas, in reality, the allowed WC space strictly coming from a single model is 


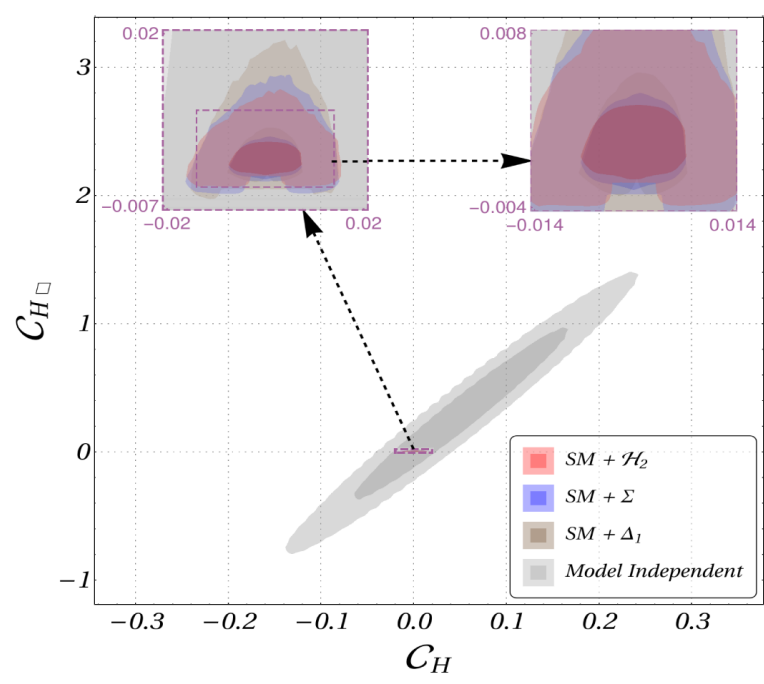

(a) $\mathcal{C}_{H}-\mathcal{C}_{H \square}$

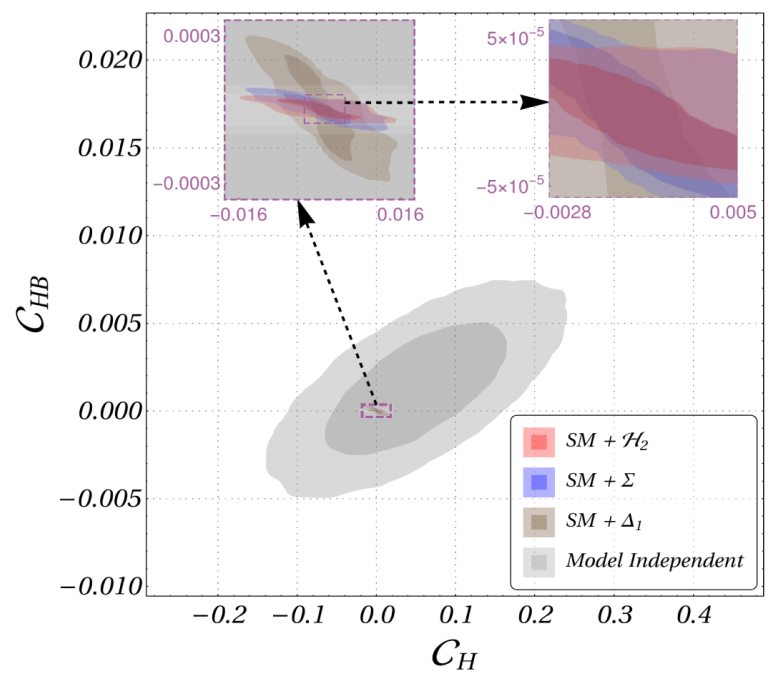

(c) $\mathcal{C}_{H}-\mathcal{C}_{H B}$

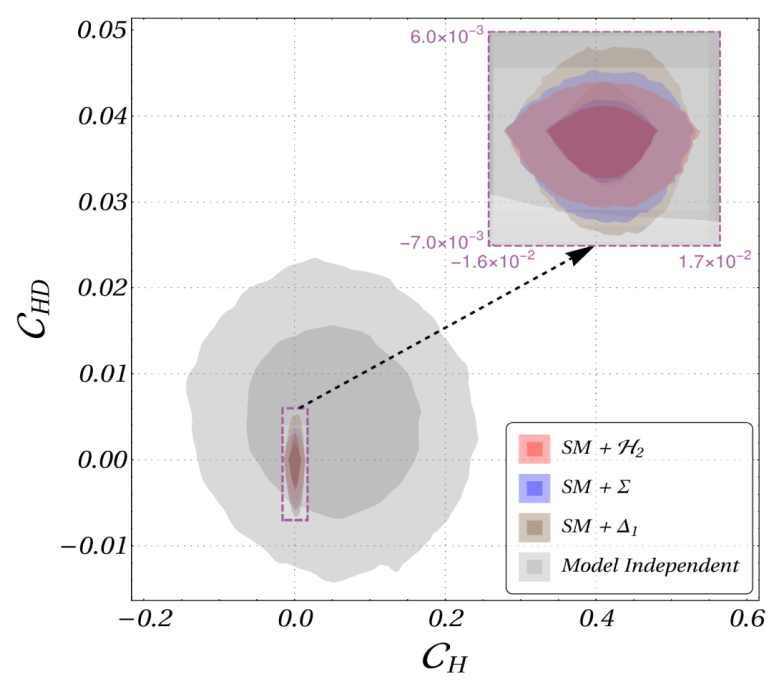

(b) $\mathcal{C}_{H}-\mathcal{C}_{H D}$

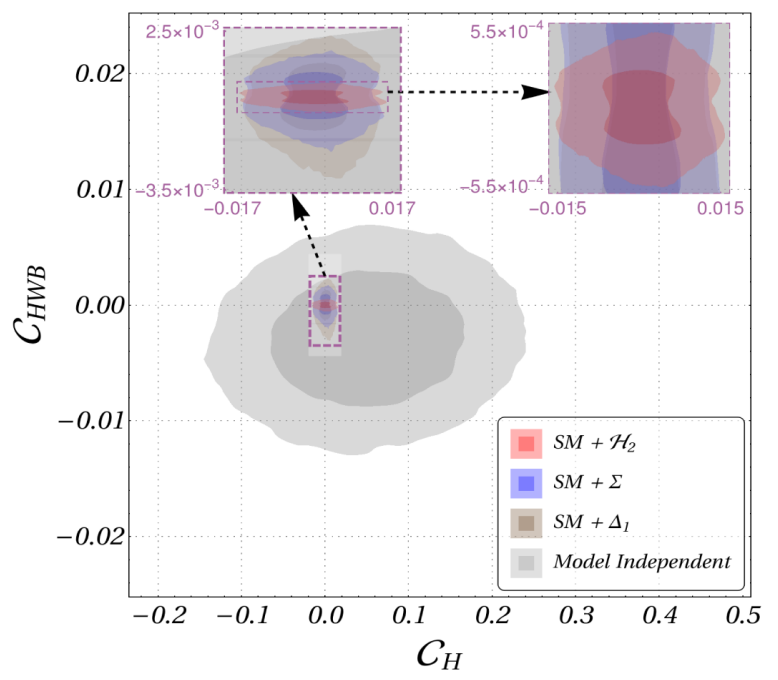

(d) $\mathcal{C}_{H}-\mathcal{C}_{H W B}$

FIG. 5. Figs. 5(a)-5(d) similar to Fig. 3 corresponding to the class of electroweak multiplet scalar scenarios, namely, $\mathrm{SM}+\Delta_{1}$, $\mathrm{SM}+\mathcal{H}_{2}$, and $\mathrm{SM}+\Sigma$. Continued in Fig. 6.

constrained in a tiny region around zero (SM). Instead of this being a quirk of one or a few models, we find this fact to be true for all BSM scenarios considered in this work.

The situation turns even worse if we consider the fact that we are only using a partial set of WCs relevant to the models in question for the model-independent results. As is the norm in the community, model-independent inferences are generally obtained with all WCs present simultaneously. As we have seen from the first column of Table III, the WC spaces become considerably larger in that case. Using such results makes our model-independent inferences overwhelmingly conservative and in essence, inaccurate.

This points us to the ominous realization that depending solely on the model-independent SMEFT fit results to infer parameter spaces of individual BSM scenarios, is in fact, far from ideal. The results of this analysis motivate us to propose that during any consequential data-driven analysis of BSM theories in view of the low-energy observables, the bottom-up approach of expressing the observables in terms of SMEFT WCs should go hand in hand with the top-down way of calculating those WCs in terms of the BSM model parameters to avoid erroneous, conservative, and in effect, too hopeful statistical inferences.

\section{ROLE OF THEORETICAL CONSTRAINTS}

In this section, we will show how the theoretical constraints can affect the model parameters derived in Sec. IV H. For the sake of demonstration, we consider the SM extension with $\Delta_{1}$ as an example model. We have noted the vacuum stability and unitarity bounds (see Refs. $[77,85,86])$ as 


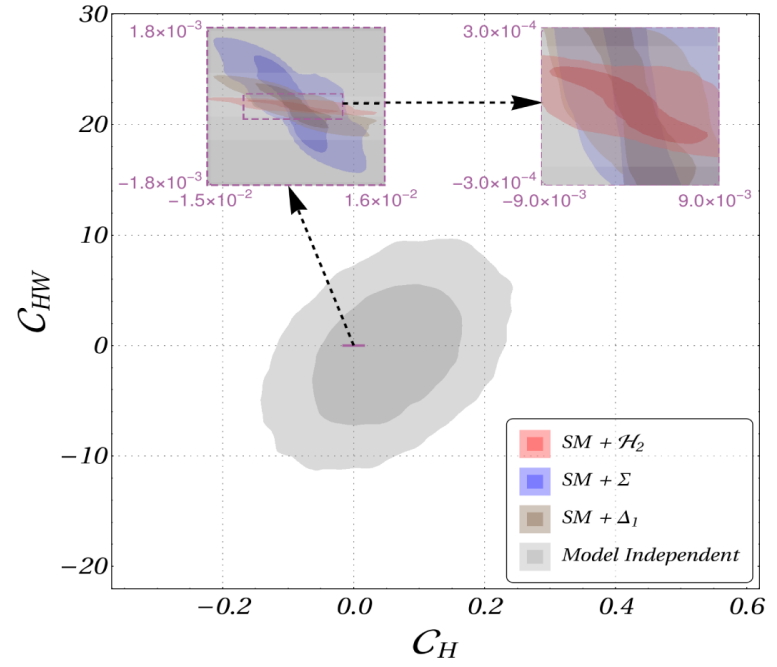

(a) $\mathcal{C}_{H}-\mathcal{C}_{H W}$

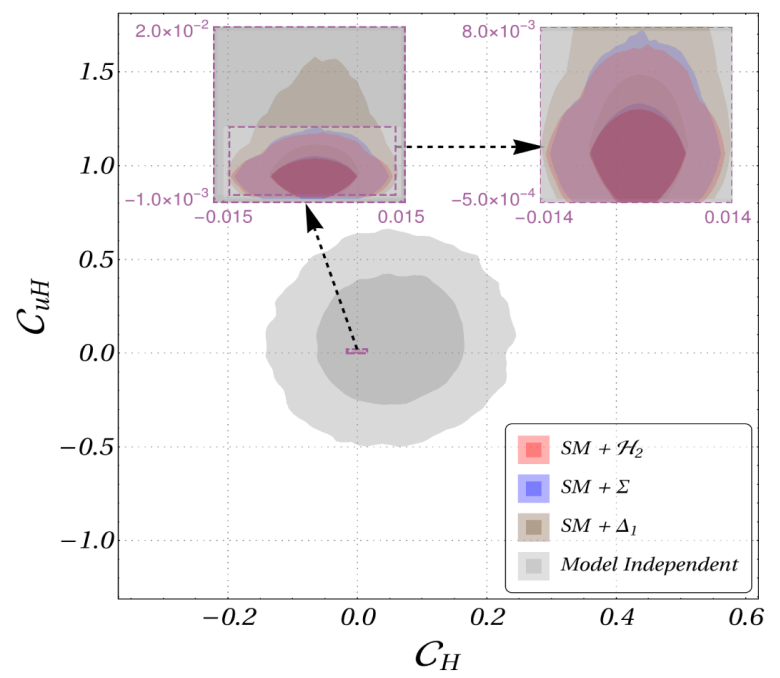

(c) $\mathcal{C}_{H}-\mathcal{C}_{u H}$

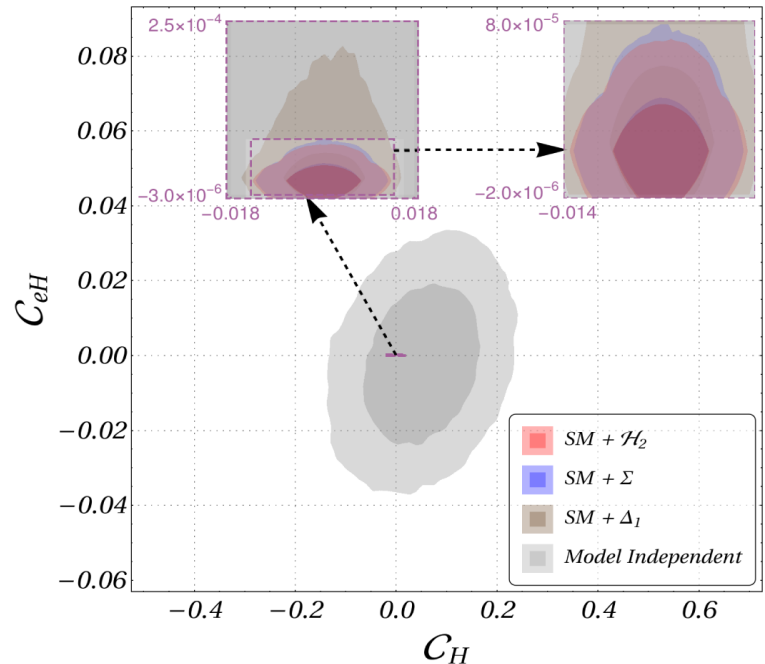

(b) $\mathcal{C}_{H}-\mathcal{C}_{e H}$

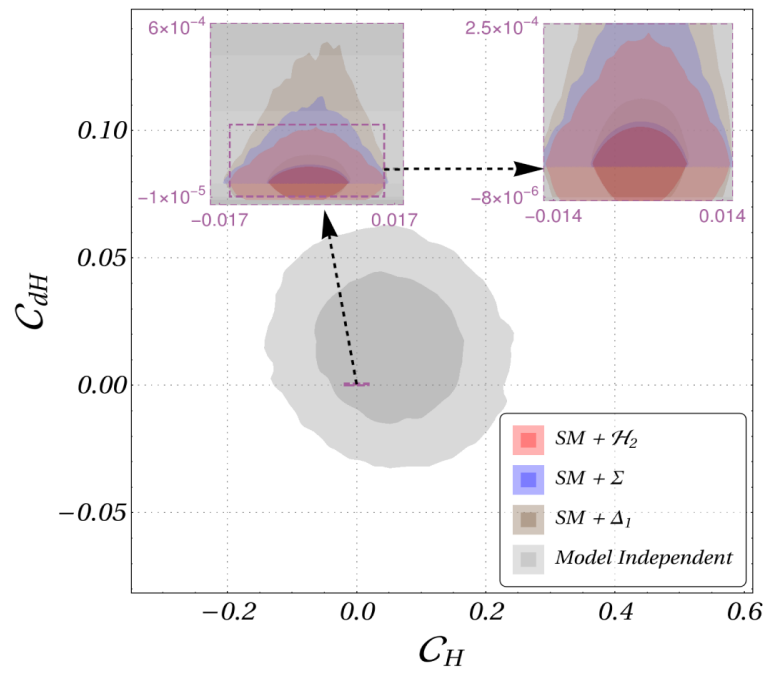

(d) $\mathcal{C}_{H}-\mathcal{C}_{d H}$

FIG. 6. Figs. 6(a)-6(d) continued from Fig. 5.

i. vacuum stability constraints

$$
\begin{aligned}
& \lambda_{\Delta_{1}, 2}+\lambda_{\Delta_{1}, 3} \geq 0, \quad \lambda_{\Delta_{1}, 2}+\frac{\lambda_{\Delta_{1}, 3}}{2} \geq 0, \quad \lambda_{\Delta_{1}, 1}+\sqrt{4 \lambda_{H}\left(\lambda_{\Delta_{1}, 2}+\lambda_{\Delta_{1}, 3}\right)} \geq 0, \\
& \lambda_{\Delta_{1}, 1}+\sqrt{4 \lambda_{H}\left(\lambda_{\Delta_{1}, 2}+\frac{\lambda_{\Delta_{1}, 3}}{2}\right)} \geq 0, \quad \lambda_{\Delta_{1}, 1}+\lambda_{\Delta_{1}, 4}+\sqrt{4 \lambda_{H}\left(\lambda_{\Delta_{1}, 2}+\lambda_{\Delta_{1}, 3}\right)} \geq 0, \\
& \lambda_{\Delta_{1}, 1}+\lambda_{\Delta_{1}, 4}+\sqrt{4 \lambda_{H}\left(\lambda_{\Delta_{1}, 2}+\frac{\lambda_{\Delta_{1}, 3}}{2}\right)} \geq 0,
\end{aligned}
$$




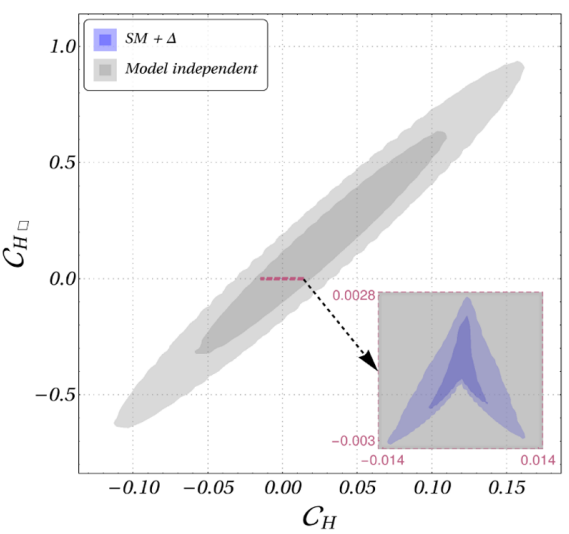

(a) $\mathcal{C}_{H}-\mathcal{C}_{H \square}$

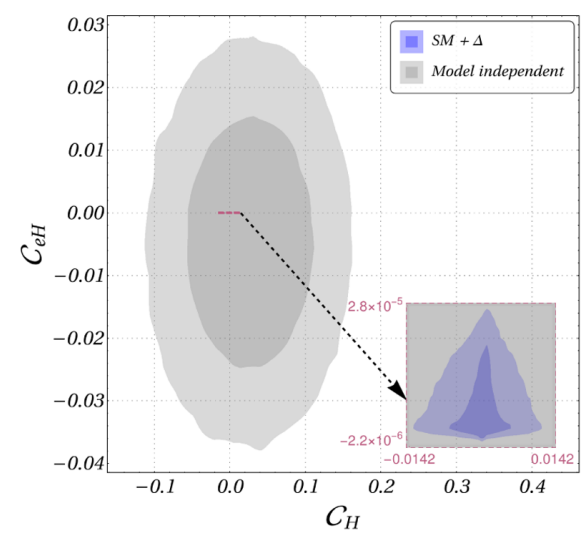

(d) $\mathcal{C}_{H}-\mathcal{C}_{e H}$

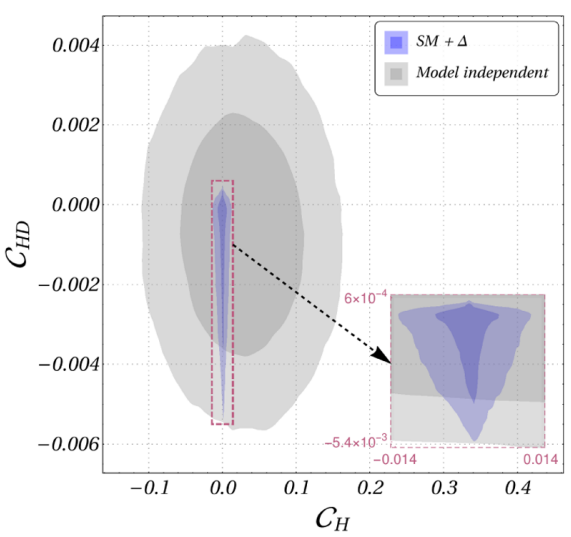

(b) $\mathcal{C}_{H}-\mathcal{C}_{H D}$

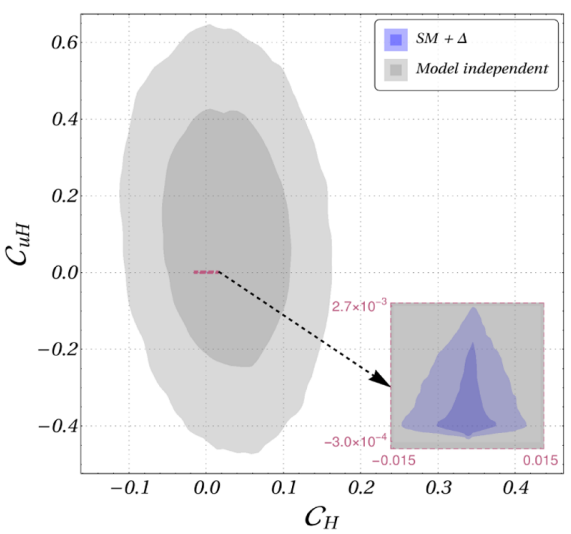

(e) $\mathcal{C}_{H}-\mathcal{C}_{u H}$

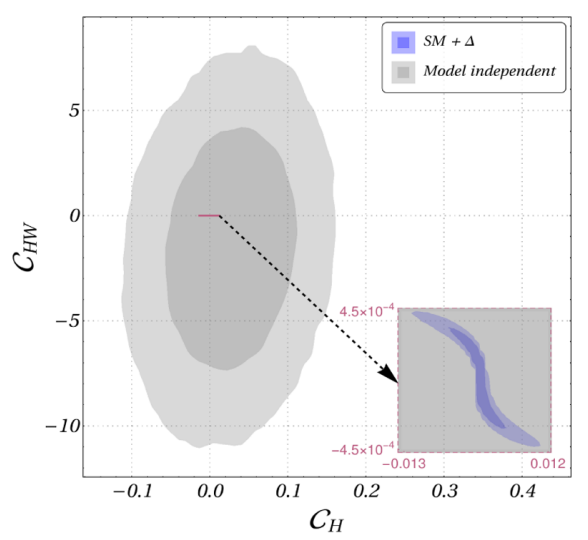

(c) $\mathcal{C}_{H}-\mathcal{C}_{H W}$

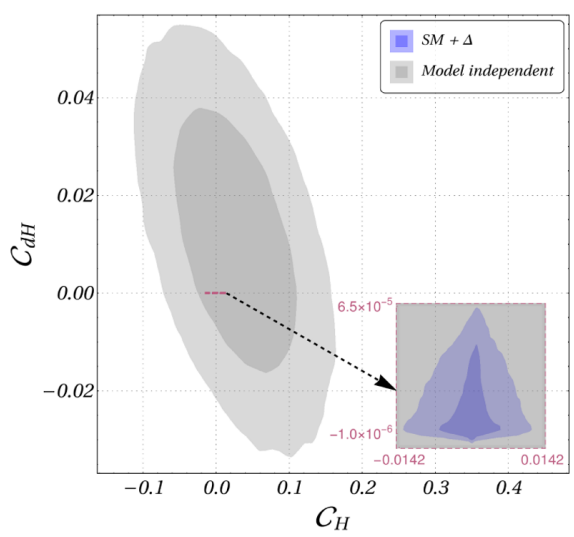

(f) $\mathcal{C}_{H}-\mathcal{C}_{d H}$

FIG. 7. Figs. 7(a)-7(f) show the comparison of the two-dimensional posteriors of model-independent WCs [gray $68 \%$ (darker) and $95 \%$ (lighter) credible intervals] with those generated from the class of isospin-triplet real scalar (SM $+\Delta)$. The blue region corresponds to the similar region obtained from the $\mathrm{SM}+\Delta$. Enlarged spaces are shown in the inset.

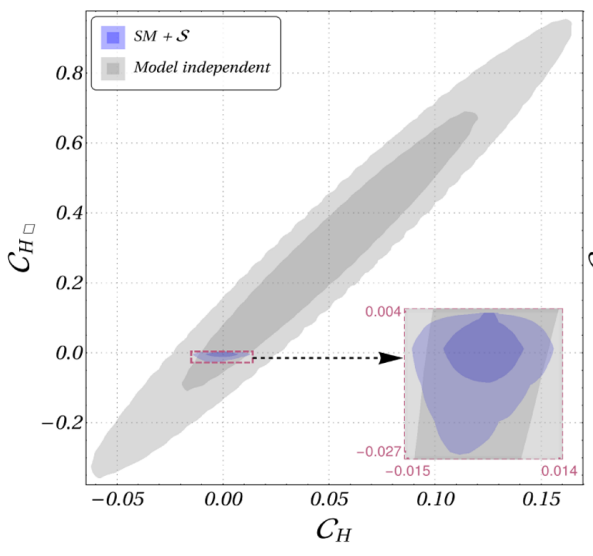

(a) $\mathcal{C}_{H}-\mathcal{C}_{H \square}$

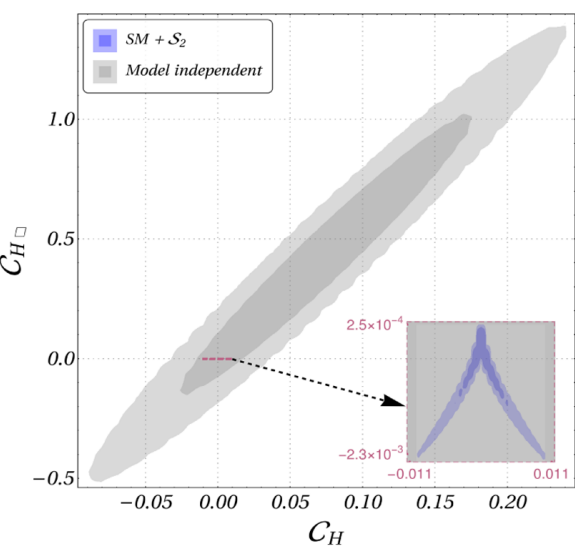

(b) $\mathcal{C}_{H}-\mathcal{C}_{H \square}$

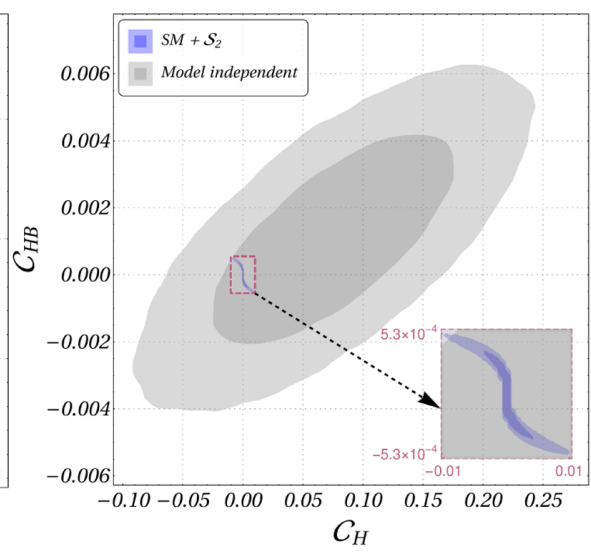

(c) $\mathcal{C}_{H}-\mathcal{C}_{H B}$

FIG. 8. Figs. 8(a)-8(c) similar to Fig. 7 corresponding to the classes of $\mathrm{SM}+\mathcal{S}$ and $\mathrm{SM}+\mathcal{S}_{2}$ with two and three mapped effective operators, respectively. 
ii. unitarity constraints

$$
\begin{array}{ll}
\lambda_{\Delta_{1}, 2}+2 \lambda_{\Delta_{1}, 3} \leq 4 \pi, & 4 \lambda_{\Delta_{1}, 2}+3 \lambda_{\Delta_{1}, 3} \leq 4 \pi, \quad 2 \lambda_{\Delta_{1}, 2}-\lambda_{\Delta_{1}, 3} \leq 8 \pi, \\
\left|\lambda_{\Delta_{1}, 1}+\lambda_{\Delta_{1}, 4}\right| \leq 8 \pi, & \left|\lambda_{\Delta_{1}, 1}\right| \leq 8 \pi, \quad\left|2 \lambda_{\Delta_{1}, 1}+3 \lambda_{\Delta_{1}, 4}\right| \leq 16 \pi, \\
\left|2 \lambda_{\Delta_{1}, 1}-\lambda_{\Delta_{1}, 4}\right| \leq 8 \pi, & \left|\lambda_{\Delta_{1}, 4}\right| \leq \min \sqrt{\left(4 \lambda_{H} \pm 16 \pi\right)\left(\lambda_{\Delta_{1}, 2}+2 \lambda_{\Delta_{1}, 3} \pm 4 \pi\right)}, \\
\left|2 \lambda_{\Delta_{1}, 1}+\lambda_{\Delta_{1}, 4}\right| & \leq \sqrt{2\left(4 \lambda_{H}-\frac{16}{3} \pi\right)\left(4 \lambda_{\Delta_{1}, 2}+3 \lambda_{\Delta_{1}, 3}-4 \pi\right) .}
\end{array}
$$

Here, $\lambda_{H}=\frac{m_{H}^{2}}{2 v^{2}}$ is the SM Higgs self-quartic coupling.

The marginalized two-dimensional posterior distribution from the Bayesian fit for parameters $\lambda_{\Delta_{1}, 1}$ and $\lambda_{\Delta_{1}, 4}$ enclosing the $68 \%$ and $95 \%$ probability regions is shown in Fig. 9. The unitary and vacuum stability bounds stated in Eqs. (6.1) and (6.2) are also shown in the figure. The crosshatched region denotes the parameter space disallowed by the theoretical bounds with $\lambda_{\Delta_{1}, 2}=\lambda_{\Delta_{1}, 3}=0$. This shows that the allowed parameter space consistent with experimental data may not be compatible with the theoretical constraints for any arbitrary choice of other parameters of the model. Thus, before performing the phenomenological analysis while choosing the benchmark values for the parameters, the inferred parameter space must be checked against the theoretical constraints.

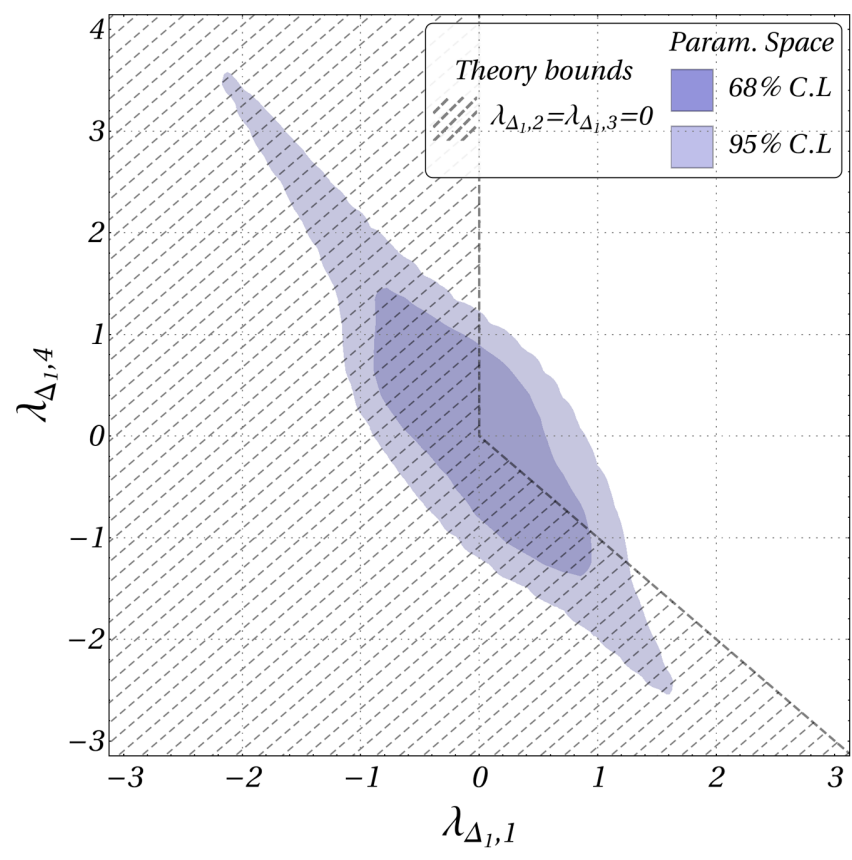

FIG. 9. The marginalized 2D posterior for the $\lambda_{\Delta_{1}, 1}$ and $\lambda_{\Delta_{1}, 4}$ for the $\mathrm{SM}+\Delta_{1}$ extension. The crosshatched region denotes the parameter space disallowed by the theoretical bounds for $\lambda_{\Delta_{1}, 2}=\lambda_{\Delta_{1}, 3}=0$.

\section{CONCLUSION AND REMARKS}

For long, experimental observations have persuaded us to propose numerous theoretically consistent BSMs with widely varying underlying symmetries and particle content. The unsettling aspect of the phenomenological landscape is that, on one hand, this diverse group of BSM scenarios, often proposed to address similar queries, is not viable for scrutiny under the same microscope. On the other hand, to understand the correct nature of new physics, it is necessary to find a common ground for multiple BSM scenarios, from where we can start making comparative remarks about them. The resolution of this apparent conflict has been our chief motivation for the present work.

To achieve this, we proceed in this work to rerealize the minimal extensions of the SM with the help of EFT. As the experimental touchstone, we define our set of observables using the EWPOs and the Higgs signal strengths from RunI and -II CMS and ATLAS data. Noting that the complete set of our adopted observables can be recast in terms of 18 SMEFT dimension-six operators, we first estimate the Bayesian posteriors of the respective 18 WCs (both taken together and individually), assuming them to be independent. To estimate the WC spaces and their correlations, we use the marginalized one- and two-dimensional posteriors of those WCs.

On the model side, we consider 11 BSM scenarios, each of them an extension of the SM by a single heavy scalar multiplet. We integrate out these heavy fields and compute the effective operators and associated WCs up to one-loop level, thus making the WCs correlated through the BSM parameters. While computing the WCs, we ignore the heavy-light mixing in the loop.

Further noticing that only ten of the 18 operators can be generated from the said BSM scenarios, we recreate the statistical analysis considering the relevant ten WCs independent. Comparing the marginal posteriors of each WC present in both ten and 18 WCs fits, we observe that simultaneous fits of a larger number of independent WCs nontrivially increase the allowed parameter space of each WC. Based on this variation of the allowed WC space, together with the observation that some of the scenarios 
lead to the same set of effective dimension-six operators, we categorize such BSMs to form six classes encapsulating 11 models, with each class containing a distinct set of WCs. The estimates of these groups of WCs constitute the modelindependent part of our analysis.

Next, we perform a Bayesian analysis to estimate the ranges of the parameters appearing in the Lagrangians of all 11 BSM scenarios. Using samples from these posteriors, we then reconstruct the WCs of the class to which those models belong. This enables us to display and compare the ranges and correlations of the WCs coming from different models of the same class to their respective modelindependent estimates through two-dimensional marginal distributions in the WC space. We also show, with an example model, how the theoretical constraints, e.g., vacuum stability and unitarity, can play a further crucial role to rule out some of the BSM parameter space which is consistent with the experimental data. The numerical results of the entire analysis, along with all figures (including those not added in the draft) are available in the GitHub repository [39] associated with this work.

This method of employing EFT and using the common WC spaces shared by BSM scenarios provides a platform to compare apparently disconnected UV theories described by the same IR d.o.f. respecting the same symmetry, and paves the way toward a complete data-driven way of addressing the intractable inverse problem. Though EFT cannot replace the full theoretical computation, it can help us sniff out the correct nature of NP. It can be further used to understand the underlying degeneracy in model space, with a clue to break the same degeneracy including more observables. This approach can be replicated even in the event of the discovery of a new BSM particle. In that case, we need to compute the complete set of effective operators for the new theory (BSMEFT $[87,88]$ ) and recast the full observable set in terms of these new operators. With the help of GrIP [89], CODEx [37], and an increasing number of observables from different sectors, a suitable statistical inference process could, hopefully, unveil the correct nature of new theories.

\section{ACKNOWLEDGMENTS}

The works of A., S. D. B., and J. C. are supported by the Science and Engineering Research Board, Government of India, under the Grant Agreements No. SERB/PHY/ 2016348 (Early Career Research Grant) and No. SERB/ PHY/2019501 (MATRICS) and an Initiation Research Grant Agreement No. IITK/PHY/2015077 by IIT Kanpur.

\section{APPENDIX A: SM FIT OF ELECTROWEAK PRECISION OBSERVABLES}

Using the experimental inputs and theoretical predictions of the electroweak precision observables mentioned in Sec. II A, we perform the SM electroweak fit in terms of five parameters using the Bayesian framework. The uniform priors are chosen with ranges of $\{90,92\},\{120,130\}$, $\{170,180\},\{0,0.2\}$, and $\{0.02,0.03\}$ for the SM parameters mass of the $Z$ boson $\left(m_{Z}\right)$, mass of the Higgs boson $\left(m_{H}\right)$, mass of the top quark $\left(m_{t}\right)$, strong coupling constant $\left[\alpha_{s}\left(m_{Z}^{2}\right)\right]$, and the hadronic contribution to the running of $\alpha$ $\left[\Delta \alpha_{\text {had }}^{(5)}\left(m_{Z}^{2}\right)\right]$, respectively. The central tendencies and dispersions of the parameters obtained after performing a Bayesian fit in terms of 19 observables are given in the second and third columns of Table XIII.

The obtained results are cross-checked and found to agree with the global electroweak fit performed by the Gfitter group [90]. These fitted SM parameters are considered as nuisance parameters in the main part of our analysis, and these results are fed into the SMEFT fits as multinormal priors.

TABLE XIII. Results of the SM fit of EWPO.

\begin{tabular}{|c|c|c|c|c|c|c|}
\hline \multirow{2}{*}{$\frac{\text { Parameters }}{m_{Z}(\mathrm{GeV})}$} & \multirow{2}{*}{$\frac{\text { Fit values }}{91.188 \pm 0.002}$} & \multicolumn{5}{|c|}{ Correlations } \\
\hline & & 1 & 0.002 & -0.097 & -0.007 & 0.040 \\
\hline$m_{H}(\mathrm{GeV})$ & $125.1 \pm 0.2$ & & 1 & 0.002 & -0.001 & -0.001 \\
\hline$m_{t}(\mathrm{GeV})$ & $173.554 \pm 0.843$ & & & 1 & 0.045 & 0.098 \\
\hline$\alpha_{s}$ & $0.118 \pm 0.003$ & & & & 1 & 0.010 \\
\hline$\Delta \alpha_{\text {had }}^{(5)}\left(m_{Z}^{2}\right)$ & $0.0276 \pm 0.0001$ & & & & & 1 \\
\hline
\end{tabular}




\section{APPENDIX B: CORRELATIONS FOR THE 18 WCs AND TEN WCs FIT}

TABLE XIV. Correlations among the 18 WCs with the fit results shown in column 2 of Table III.

\begin{tabular}{|c|c|c|c|c|c|c|c|c|c|c|c|c|c|c|c|c|c|c|}
\hline \multirow[b]{2}{*}{ WCs } & \multicolumn{18}{|c|}{ Correlations } \\
\hline & $\mathcal{C}_{H}$ & $\mathcal{C}_{H \square}$ & $\mathcal{C}_{H D}$ & $\mathcal{C}_{H B}$ & $\mathcal{C}_{H W}$ & $\mathcal{C}_{H W B}$ & $\mathcal{C}_{H G}$ & $\mathcal{C}_{e H}$ & $\mathcal{C}_{u H}$ & $\mathcal{C}_{d H}$ & $\mathcal{C}_{H l}^{(1)}$ & $\mathcal{C}_{H l}^{(3)}$ & $\mathcal{C}_{H q}^{(1)}$ & $\mathcal{C}_{H q}^{(3)}$ & $\mathcal{C}_{\mathrm{He}}$ & $\mathcal{C}_{H u}$ & $\mathcal{C}_{H d}$ & $\mathcal{C}_{l l}$ \\
\hline$\overline{\mathcal{C}_{H}}$ & 1 & 0.998 & -0.787 & 0.637 & 0.619 & 0.786 & -0.281 & 0.170 & -0.006 & -0.269 & -0.574 & -0.784 & 0.048 & -0.770 & -0.730 & 0.024 & 0.018 & -0.757 \\
\hline $\mathcal{C}_{H \square}$ & & 1 & -0.767 & 0.649 & 0.618 & 0.767 & -0.278 & 0.178 & -0.005 & -0.261 & -0.559 & -0.765 & 0.048 & -0.750 & -0.712 & 0.023 & 0.018 & -0.738 \\
\hline $\mathcal{C}_{H D}$ & & & 1 & -0.289 & -0.525 & -0.999 & 0.292 & -0.004 & 0.029 & 0.358 & 0.733 & 0.997 & -0.048 & 0.975 & 0.929 & -0.015 & -0.026 & 0.960 \\
\hline $\mathcal{C}_{H B}$ & & & & 1 & 0.501 & 0.289 & -0.181 & 0.350 & 0.148 & 0.152 & -0.210 & -0.287 & 0.019 & -0.278 & -0.265 & -0.003 & 0.022 & -0.277 \\
\hline $\mathcal{C}_{H W}$ & & & & & 1 & 0.525 & -0.202 & 0.165 & 0.124 & -0.044 & -0.387 & -0.522 & 0.006 & -0.498 & -0.485 & -0.047 & 0.055 & -0.503 \\
\hline $\mathcal{C}_{H W B}$ & & & & & & 1 & -0.292 & 0.004 & -0.029 & -0.358 & -0.734 & -0.997 & 0.049 & -0.975 & -0.929 & 0.015 & 0.026 & -0.960 \\
\hline $\mathcal{C}_{H G}$ & & & & & & & 1 & -0.317 & 0.206 & -0.645 & 0.218 & 0.293 & -0.013 & 0.287 & 0.278 & -0.011 & -0.009 & 0.281 \\
\hline $\mathcal{C}_{e H}$ & & & & & & & & 1 & -0.010 & 0.355 & -0.007 & -0.005 & 0.015 & -0.004 & -0.008 & 0.010 & 0.020 & -0.004 \\
\hline $\mathcal{C}_{u H}$ & & & & & & & & & 1 & -0.019 & 0.030 & 0.037 & 0.002 & 0.035 & 0.049 & -0.015 & -0.008 & 0.033 \\
\hline $\mathcal{C}_{d H}$ & & & & & & & & & & 1 & 0.258 & 0.356 & -0.024 & 0.351 & 0.329 & -0.015 & 0.003 & 0.344 \\
\hline $\mathcal{C}_{H I}^{(1)}$ & & & & & & & & & & & 1 & 0.704 & -0.048 & 0.726 & 0.787 & 0 & -0.061 & 0.560 \\
\hline $\mathcal{C}_{H I}^{(3)}$ & & & & & & & & & & & & 1 & -0.050 & 0.974 & 0.934 & -0.014 & -0.030 & 0.977 \\
\hline $\mathcal{C}_{H a}^{(1)}$ & & & & & & & & & & & & & 1 & -0.143 & -0.068 & 0.649 & 0.351 & -0.050 \\
\hline $\mathcal{C}_{H a q}^{(3)}$ & & & & & & & & & & & & & & 1 & 0.902 & -0.194 & 0.101 & 0.934 \\
\hline $\mathcal{C}_{\mathrm{He}}^{\mathrm{Hq}}$ & & & & & & & & & & & & & & & 1 & 0.005 & -0.101 & 0.897 \\
\hline $\mathcal{C}_{H u}$ & & & & & & & & & & & & & & & & 1 & -0.137 & -0.009 \\
\hline $\mathcal{C}_{H d}$ & & & & & & & & & & & & & & & & & 1 & -0.043 \\
\hline $\mathcal{C}_{l l}$ & & & & & & & & & & & & & & & & & & 1 \\
\hline
\end{tabular}

TABLE XV. Correlations among the ten WCs with the fit results shown in column 4 of Table III.

\begin{tabular}{|c|c|c|c|c|c|c|c|c|c|c|}
\hline \multirow[b]{2}{*}{ WCs } & \multicolumn{10}{|c|}{ Correlations } \\
\hline & $\mathcal{C}_{H}$ & $\mathcal{C}_{H \square}$ & $\mathcal{C}_{H D}$ & $\mathcal{C}_{H B}$ & $\mathcal{C}_{H W}$ & $\mathcal{C}_{H W B}$ & $\mathcal{C}_{H G}$ & $\mathcal{C}_{e H}$ & $\mathcal{C}_{u H}$ & $\mathcal{C}_{d H}$ \\
\hline $\mathcal{C}_{H}$ & 1 & 0.996 & -0.065 & 0.698 & 0.402 & 0.063 & -0.077 & 0.272 & 0.037 & 0.016 \\
\hline $\mathcal{C}_{H \square}$ & & 1 & -0.061 & 0.700 & 0.403 & 0.0597 & -0.078 & 0.273 & 0.036 & 0.016 \\
\hline $\mathcal{C}_{H D}$ & & & 1 & -0.040 & -0.065 & -0.964 & -0.020 & 0.002 & -0.157 & 0.028 \\
\hline $\mathcal{C}_{H B}$ & & & & 1 & 0.434 & 0.047 & -0.097 & 0.366 & 0.165 & 0.280 \\
\hline $\mathcal{C}_{H W}$ & & & & & 1 & 0.070 & -0.053 & 0.190 & 0.169 & 0.177 \\
\hline $\mathcal{C}_{H W B}$ & & & & & & 1 & 0.030 & -0.002 & 0.198 & -0.028 \\
\hline $\mathcal{C}_{H G}$ & & & & & & & 1 & -0.315 & 0.203 & -0.836 \\
\hline $\mathcal{C}_{e H}$ & & & & & & & & 1 & -0.010 & 0.369 \\
\hline $\mathcal{C}_{u H}$ & & & & & & & & & 1 & -0.030 \\
\hline $\mathcal{C}_{d H}$ & & & & & & & & & & 1 \\
\hline
\end{tabular}

[1] W. Buchmuller and D. Wyler, Effective Lagrangian analysis of new interactions and flavor conservation, Nucl. Phys. B268, 621 (1986).

[2] B. Grzadkowski, M. Iskrzynski, M. Misiak, and J. Rosiek, Dimension-six terms in the Standard Model Lagrangian, J. High Energy Phys. 10 (2010) 085.

[3] J. Elias-Miro, J. Espinosa, E. Masso, and A. Pomarol, Higgs windows to new physics through $d=6$ operators: Constraints and one-loop anomalous dimensions, J. High Energy Phys. 11 (2013) 066.
[4] F. del Aguila and J. de Blas, Electroweak constraints on new physics, Fortschr. Phys. 59, 1036 (2011).

[5] G. Giudice, C. Grojean, A. Pomarol, and R. Rattazzi, The strongly-interacting light Higgs, J. High Energy Phys. 06 (2007) 045.

[6] M. Ciuchini, E. Franco, S. Mishima, and L. Silvestrini, Electroweak precision observables, new physics and the nature of a 126 GeV Higgs boson, J. High Energy Phys. 08 (2013) 106.

[7] J. Ellis, V. Sanz, and T. You, The effective Standard Model after LHC Run I, J. High Energy Phys. 03 (2015) 157. 
[8] A. Falkowski and F. Riva, Model-independent precision constraints on dimension-6 operators, J. High Energy Phys. 02 (2015) 039.

[9] V. Cirigliano, W. Dekens, J. de Vries, and E. Mereghetti, Constraining the top-Higgs sector of the standard model effective field theory, Phys. Rev. D 94, 034031 (2016).

[10] S. Jana and S. Nandi, New physics scale from Higgs observables with effective dimension-6 operators, Phys. Lett. B 783, 51 (2018).

[11] L. Berthier and M. Trott, Towards consistent electroweak precision data constraints in the SMEFT, J. High Energy Phys. 05 (2015) 024.

[12] C. W. Murphy, Statistical approach to Higgs boson couplings in the standard model effective field theory, Phys. Rev. D 97, 015007 (2018).

[13] M. Ciuchini, E. Franco, S. Mishima, M. Pierini, L. Reina, and L. Silvestrini, Update of the electroweak precision fit, interplay with Higgs-boson signal strengths and modelindependent constraints on new physics, Nucl. Part. Phys. Proc. 273-275, 2219 (2016).

[14] J. Haller, A. Hoecker, R. Kogler, K. Mnig, T. Peiffer, and J. Stelzer, Update of the global electroweak fit and constraints on two-Higgs-doublet models, Eur. Phys. J. C 78, 675 (2018).

[15] J. Ellis, C. W. Murphy, V. Sanz, and T. You, Updated global SMEFT fit to Higgs, diboson and electroweak data, J. High Energy Phys. 06 (2018) 146.

[16] J. de Blas, M. Ciuchini, E. Franco, S. Mishima, M. Pierini, L. Reina, and L. Silvestrini, Electroweak precision observables and Higgs-boson signal strengths in the standard model and beyond: Present and future, J. High Energy Phys. 12 (2016) 135.

[17] J. Ellis, C. W. Murphy, V. Sanz, and T. You, Updated global SMEFT fit to Higgs, diboson and electroweak data, J. High Energy Phys. 06 (2018) 146.

[18] E. da Silva Almeida, A. Alves, N. Rosa Agostinho, O. J. boli, and M. Gonzalez-Garcia, Electroweak sector under scrutiny: A combined analysis of LHC and electroweak precision data, Phys. Rev. D 99, 033001 (2019).

[19] B. Dumont, S. Fichet, and G. von Gersdorff, A Bayesian view of the Higgs sector with higher dimensional operators, J. High Energy Phys. 07 (2013) 065.

[20] S. van Beek, E. R. Nocera, J. Rojo, and E. Slade, Constraining the SMEFT with Bayesian reweighting, SciPost Phys. 7, 070 (2019).

[21] N. Sato, J. Owens, and H. Prosper, Bayesian reweighting for global fits, Phys. Rev. D 89, 114020 (2014).

[22] N. Castro, J. Erdmann, C. Grunwald, K. Kröninger, and N.-A. Rosien, EFTfitter-A tool for interpreting measurements in the context of effective field theories, Eur. Phys. J. C 76, 432 (2016).

[23] B. Henning, X. Lu, and H. Murayama, How to use the Standard Model effective field theory, J. High Energy Phys. 01 (2016) 023.

[24] A. Drozd, J. Ellis, J. Quevillon, and T. You, The universal one-loop effective action, J. High Energy Phys. 03 (2016) 180.

[25] B. Henning, X. Lu, and H. Murayama, One-loop matching and running with covariant derivative expansion, J. High Energy Phys. 01 (2018) 123.
[26] S. A. R. Ellis, J. Quevillon, T. You, and Z. Zhang, Mixed heavylight matching in the universal one-loop effective action, Phys. Lett. B 762, 166 (2016).

[27] J. Fuentes-Martin, J. Portoles, and P. Ruiz-Femenia, Integrating out heavy particles with functional methods: A simplified framework, J. High Energy Phys. 09 (2016) 156.

[28] Z. Zhang, Covariant diagrams for one-loop matching, J. High Energy Phys. 05 (2017) 152.

[29] S. A. R. Ellis, J. Quevillon, T. You, and Z. Zhang, Extending the universal one-loop effective action: Heavy-light coefficients, J. High Energy Phys. 08 (2017) 054.

[30] J. de Blas, J. C. Criado, M. Prez-Victoria, and J. Santiago, Effective description of general extensions of the standard model: The complete tree-level dictionary, J. High Energy Phys. 03 (2018) 109.

[31] M. Krmer, B. Summ, and A. Voigt, Completing the scalar and fermionic universal one-loop effective action, J. High Energy Phys. 01 (2020) 079.

[32] U. Haisch, M. Ruhdorfer, E. Salvioni, E. Venturini, and A. Weiler, Singlet night in Feynman-Ville: One-loop matching of a real scalar, J. High Energy Phys. 04 (2020) 164.

[33] M. Gorbahn, J. M. No, and V. Sanz, Benchmarks for Higgs effective theory: Extended Higgs sectors, J. High Energy Phys. 10 (2015) 036.

[34] S. Dawson, S. Homiller, and S. D. Lane, Putting SMEFT fits to work, Phys. Rev. D 102, 055012 (2020).

[35] A. Drozd, J. Ellis, J. Quevillon, and T. You, Comparing EFT and exact one-loop analyses of non-degenerate stops, J. High Energy Phys. 06 (2015) 028.

[36] S. Das Bakshi, J. Chakrabortty, C. Englert, M. Spannowsky, and P. Stylianou, ATLAS violating $C P$ effectively, arXiv: 2009.13394.

[37] S. Das Bakshi, J. Chakrabortty, and S. K. Patra, CodEx: Wilson coefficient calculator connecting SMEFT to UV theory, Eur. Phys. J. C 79, 21 (2019).

[38] S. K. Patra, optEx, 2019 (still under development; updates will be available online in the future).

[39] See Supplemental Material at http://link.aps.org/ supplemental/10.1103/PhysRevD.103.076007 for more details.

[40] S. Schael et al. (ALEPH, DELPHI, L3, OPAL, SLD, LEP Electroweak Working Group, SLD Electroweak Group, and SLD Heavy Flavour Group Collaborations), Precision electroweak measurements on the $Z$ resonance, Phys. Rep. 427, 257 (2006).

[41] M. Tanabashi et al. (Particle Data Group Collaboration), Review of particle physics, Phys. Rev. D 98, 030001 (2018).

[42] M. Awramik, M. Czakon, and A. Freitas, Electroweak twoloop corrections to the effective weak mixing angle, J. High Energy Phys. 11 (2006) 048.

[43] A. Freitas, Higher-order electroweak corrections to the partial widths and branching ratios of the $Z$ boson, J. High Energy Phys. 04 (2014) 070.

[44] H. Flacher, M. Goebel, J. Haller, A. Hocker, K. Monig, and J. Stelzer, Revisiting the global electroweak fit of the Standard Model and beyond with Gfitter, Eur. Phys. J. C 60, 543 (2009); Erratum, Eur. Phys. J. C 71, 1718 (2011).

[45] M. Awramik, M. Czakon, A. Freitas, and G. Weiglein, Precise prediction for the $W$ boson mass in the standard model, Phys. Rev. D 69, 053006 (2004). 
[46] G.-C. Cho, K. Hagiwara, Y. Matsumoto, and D. Nomura, The MSSM confronts the precision electroweak data and the muon $g-2$, J. High Energy Phys. 11 (2011) 068.

[47] G. Aad et al. (ATLAS and CMS Collaborations), Measurements of the Higgs boson production and decay rates and constraints on its couplings from a combined ATLAS and CMS analysis of the LHC $p p$ collision data at $\sqrt{s}=7$ and $8 \mathrm{TeV}$, J. High Energy Phys. 08 (2016) 045.

[48] G. Aad et al. (ATLAS Collaboration), Measurements of the Higgs boson production and decay rates and coupling strengths using $p p$ collision data at $\sqrt{s}=7$ and $8 \mathrm{TeV}$ in the ATLAS experiment, Eur. Phys. J. C 76, 6 (2016).

[49] G. Aad et al. (ATLAS Collaboration), Higgs boson production cross-section measurements and their EFT interpretation in the $4 \ell$ decay channel at $\sqrt{s}=13 \mathrm{TeV}$ with the ATLAS detector, Eur. Phys. J. C 80, 957 (2020).

[50] G. Aad et al. (ATLAS Collaboration), A search for the $Z \gamma$ decay mode of the Higgs boson in $p p$ collisions at $\sqrt{s}=$ $13 \mathrm{TeV}$ with the ATLAS detector, Phys. Lett. B 809, 135754 (2020).

[51] ATLAS Collaboration, A search for the dimuon decay of the Standard Model Higgs boson in $p p$ collisions at $\sqrt{s}=$ $13 \mathrm{TeV}$ with the ATLAS detector, http://cds.cern.ch/record/ 2682155.

[52] ATLAS Collaboration, Measurement of the associated production of a Higgs boson decaying to $b$ quarks with a vector boson at high transverse momentum in $p p$ collisions at $\sqrt{s}=13 \mathrm{TeV}$ with the ATLAS detector, http://cds.cern .ch/record/2715063.

[53] G. Aad et al. (ATLAS Collaboration), Combined measurements of Higgs boson production and decay using up to $80 \mathrm{fb}^{-1}$ of proton-proton collision data at $\sqrt{s}=13 \mathrm{TeV}$ collected with the ATLAS experiment, Phys. Rev. D 101, 012002 (2020).

[54] M. Aaboud et al. (ATLAS Collaboration), Evidence for the associated production of the Higgs boson and a top quark pair with the ATLAS detector, Phys. Rev. D 97, 072003 (2018).

[55] M. Aaboud et al. (ATLAS Collaboration), Observation of Higgs boson production in association with a top quark pair at the LHC with the ATLAS detector, Phys. Lett. B 784, 173 (2018).

[56] G. Aad et al. (ATLAS Collaboration), Measurement of the production cross section for a Higgs boson in association with a vector boson in the $H \rightarrow W W^{*} \rightarrow \ell \nu \ell \nu$ channel in $p p$ collisions at $\sqrt{s}=13 \mathrm{TeV}$ with the ATLAS detector, Phys. Lett. B 798, 134949 (2019).

[57] A. M. Sirunyan et al. (CMS Collaboration), Combined measurements of Higgs boson couplings in proton-proton collisions at $\sqrt{s}=13 \mathrm{TeV}$, Eur. Phys. J. C 79, 421 (2019).

[58] A. M. Sirunyan et al. (CMS Collaboration), A search for the standard model Higgs boson decaying to charm quarks, J. High Energy Phys. 03 (2020) 131.

[59] I. Brivio and M. Trott, The Standard Model as an effective field theory, Phys. Rep. 793, 1 (2019).

[60] R. Alonso, E. E. Jenkins, A. V. Manohar, and M. Trott, Renormalization group evolution of the Standard Model dimension six operators III: Gauge coupling dependence and phenomenology, J. High Energy Phys. 04 (2014) 159.
[61] S. Dawson and P. P. Giardino, Electroweak and QCD corrections to $Z$ and $W$ pole observables in the standard model EFT, Phys. Rev. D 101, 013001 (2020).

[62] C. W. Murphy, Statistical approach to Higgs boson couplings in the standard model effective field theory, Phys. Rev. D 97, 015007 (2018).

[63] W. K. Hastings, Monte Carlo sampling methods using Markov chains and their applications, Biometrika 57, 97 (1970).

[64] A.E. Raftery and S.M. Lewis, Practical Markov chain Monte Carlo: Comment: One long run with diagnostics: Implementation strategies for Markov chain Monte Carlo, Stat. Sci. 7, 493 (1992).

[65] R. D. Cook, Detection of influential observation in linear regression, Technometrics 19, 15 (1977).

[66] R. D. Cook, Influential observations in linear regression, J. Am. Stat. Assoc. 74, 169 (1979).

[67] K. A. Bollen and R. W. Jackman, Regression diagnostics: An expository treatment of outliers and influential cases, Socio. Methods Res. 13, 510 (1985).

[68] M. Jiang, N. Craig, Y.-Y. Li, and D. Sutherland, Complete one-loop matching for a singlet scalar in the Standard Model EFT, J. High Energy Phys. 02 (2019) 031.

[69] S. Dawson and C.W. Murphy, Standard Model EFT and extended scalar sectors, Phys. Rev. D 96, 015041 (2017).

[70] U. Haisch, M. Ruhdorfer, E. Salvioni, E. Venturini, and A. Weiler, Singlet night in Feynman-Ville: One-loop matching of a real scalar, J. High Energy Phys. 04 (2020) 164; Erratum, J. High Energy Phys.07 (2020) 066.

[71] J. de Blas, M. Chala, M. Perez-Victoria, and J. Santiago, Observable effects of general new scalar particles, J. High Energy Phys. 04 (2015) 078.

[72] J. de Blas, J. Criado, M. Perez-Victoria, and J. Santiago, Effective description of general extensions of the Standard Model: The complete tree-level dictionary, J. High Energy Phys. 03 (2018) 109.

[73] N. G. Deshpande and E. Ma, Pattern of symmetry breaking with two Higgs doublets, Phys. Rev. D 18, 2574 (1978).

[74] S. Nie and M. Sher, Vacuum stability bounds in the twoHiggs doublet model, Phys. Lett. B 449, 89 (1999).

[75] V. Branchina, F. Contino, and P. Ferreira, Electroweak vacuum lifetime in two Higgs doublet models, J. High Energy Phys. 11 (2018) 107.

[76] A. Pilaftsis and C. E. Wagner, Higgs bosons in the minimal supersymmetric standard model with explicit $C P$ violation, Nucl. Phys. B553, 3 (1999).

[77] A. Arhrib, R. Benbrik, M. Chabab, G. Moultaka, M. Peyranere, L. Rahili, and J. Ramadan, The Higgs potential in the type II seesaw model, Phys. Rev. D 84, 095005 (2011).

[78] K. S. Babu, S. Nandi, and Z. Tavartkiladze, New mechanism for neutrino mass generation and triply charged Higgs bosons at the LHC, Phys. Rev. D 80, 071702 (2009).

[79] G. Bambhaniya, J. Chakrabortty, S. Goswami, and P. Konar, Generation of neutrino mass from new physics at $\mathrm{TeV}$ scale and multilepton signatures at the LHC, Phys. Rev. D 88, 075006 (2013). 
[80] M. Bauer and M. Neubert, Minimal Leptoquark Explanation for the $R_{D^{(*)}}, R_{K}$, and $(g-2)_{g}$ Anomalies, Phys. Rev. Lett. 116, 141802 (2016).

[81] P. Bandyopadhyay and R. Mandal, Vacuum stability in an extended standard model with a leptoquark, Phys. Rev. D 95, 035007 (2017).

[82] S. Davidson and S. Descotes-Genon, Minimal flavour violation for leptoquarks, J. High Energy Phys. 11 (2010) 073.

[83] J. M. Arnold, B. Fornal, and M. B. Wise, Phenomenology of scalar leptoquarks, Phys. Rev. D 88, 035009 (2013).

[84] W. Buchmuller, R. Ruckl, and D. Wyler, Leptoquarks in lepton-Quark collisions, Phys. Lett. B 191, 442 (1987); Erratum, Phys. Lett. B 448, 320 (1999).

[85] E. J. Chun, H. M. Lee, and P. Sharma, Vacuum stability, perturbativity, EWPD and Higgs-to-diphoton rate in type II seesaw models, J. High Energy Phys. 11 (2012) 106.
[86] N. Haba, H. Ishida, N. Okada, and Y. Yamaguchi, Vacuum stability and naturalness in type-II seesaw, Eur. Phys. J. C 76, 333 (2016).

[87] U. Banerjee, J. Chakrabortty, S. Prakash, S. U. Rahaman, and M. Spannowsky, Effective operator bases for beyond Standard Model scenarios: An EFT compendium for discoveries, J. High Energy Phys. 01 (2021) 028.

[88] Anisha, S. Das Bakshi, J. Chakrabortty, and S. Prakash, Hilbert series and plethystics: Paving the path towards 2HDM- and MLRSM-EFT, J. High Energy Phys. 09 (2019) 035.

[89] U. Banerjee, J. Chakrabortty, S. Prakash, and S. U. Rahaman, Characters and group invariant polynomials of (super)fields: Road to "Lagrangian”, Eur. Phys. J. C 80, 938 (2020).

[90] Gfitter, Results of SM electroweak fit, http://project-gfitter .web.cern.ch/project-gfitter/. 